\title{
Entropy generation of tangent hyperbolic nanofluid flow over a circular cylinder in the presence of nonlinear Boussinesq approximation: A non-similar solution
}

\author{
H. Thameem Basha ${ }^{1}$ - R. Sivaraj ${ }^{1 *}$. V. \\ Ramachandra Prasad ${ }^{1}$. O.Anwar Beg ${ }^{2}$
}

Received: date / Accepted: date

\begin{abstract}
The analysis of entropy generation has received notable attention in the study of nanofluids because the prime objective of nanofluids is to admit high heat fluxes. The entropy production can be utilized to generate the entropy in any irreversible heat transfer process which is important in thermal machines. This work presents to explore the fluid transport characteristics and entropy generation of a tangent hyperbolic nanofluid over a horizontal circular cylinder with the influence of nonlinear Boussinesq approximation. The dimensionless nonlinear partial differential equations have been solved by using an implicit finite difference Keller box scheme. The impacts of active parameters on the flow field like Weissenberg number, power-law index, magnetic field, mixed convection, Brownian motion, thermal convention, thermophoresis and radiation are illustrated with graphs and tables. The current results exposed that the nanofluid velocity enhances for enhancing the mixed convection parameter. Higher values of nonlinear thermal convection parameter declines the thermal boundary thickness. Total entropy generation decreases for higher values of Eckert number. Isotherms thickness is escalated with increasing values of radiation parameter.
\end{abstract}

Keywords Tangent hyperbolic nanofluid · entropy generation · nonlinear Boussinesq approximation · inclined magnetic field · Keller box method.

R. Sivaraj

E-mail: sivaraj.kpm@gmail.com

${ }^{1}$ Department of Mathematics, School of Advanced Sciences, Vellore Institute of Technology, Vellore-632014, India

${ }^{2}$ Fluid Mechanics, Aeronautical and Mechanical Engineering Department, School of Computing, Science and Engineering, University of Salford, Manchester M54WT, UK 


\section{Nomenclature}

$a \quad$ radius of the cylinder

$B_{0} \quad$ strength of the constant magnetic field

$\mathrm{Br} \quad$ Brinkman number

$C \quad$ concentration of the fluid

$C_{f}^{*} \quad$ skin friction coefficient

$\left(C_{p}\right)_{f} \quad$ specific heat of fluid

$\mathrm{C}_{W} \quad$ concentration at the surface

$\mathrm{C}_{\infty} \quad$ ambient concentration

$D_{B} \quad$ Brownian diffusion

$D_{T} \quad$ thermophoretic diffusion

$E_{c} \quad$ Eckert number

$g \quad$ acceleration due to gravity

$k_{a} \quad$ mean absorption coefficient

$k_{f} \quad$ fluid thermal conductivity

$L_{T} \quad$ diffusion parameter

$M_{a} \quad$ magnetic parameter

$n_{a} \quad$ power law index

$N_{B} \quad$ Brownian motion parameter

$N_{c} \quad$ thermal convection parameter

$N_{G} \quad$ total entropy generation

$N_{r} \quad$ buoyancy ratio parameter

$N_{T} \quad$ thermophoresis parameters

$N u^{*} \quad$ Nusselt number

Pr Prandtl number

$R_{a} \quad$ radiation parameter

Re Reynolds number

Sc Schmidt number

$S h^{*} \quad$ Sherwood number

$S_{G} \quad$ dimensional entropy

$T$ temperature of fluid

$\mathrm{T}_{W} \quad$ temperature at the surface

$\mathrm{T}_{\infty} \quad$ ambient temperature

$u^{*}, v^{*} \quad$ velocity components in $x, y$ directions

$u_{e}^{*} \quad$ external flow velocity

$U_{\infty} \quad$ free stream velocity

$W e \quad$ Weissenberg number

$x, y \quad$ Cartesian coordinates

Greek symbols

$\alpha_{C} \quad$ concentration difference parameter

$\alpha_{T} \quad$ temperature difference parameter 
$\beta_{0} \quad$ coefficient of linear thermal expansion

$\beta_{1} \quad$ coefficient of nonlinear thermal expansion

$\Gamma \quad$ material constant

$\lambda_{c} \quad$ mixed convection parameter

$\mu_{f} \quad$ dynamic viscosity

$\rho_{f} \quad$ density of fluid

$\rho_{p} \quad$ density of particle

$\sigma \quad$ electrical conductivity

$\sigma_{B} \quad$ Stefan Boltzmann constant

$\tau \quad$ ratio between particle and base fluid

$\Psi \quad$ angle for applied magnetic field

\section{Introduction}

In recent times, the demand for optimal and efficient energy systems is increasing due to the over exploitation of energy resources. The entropy generation can be utilized to examine the efficiency of the thermodynamic performance of energy systems. The concept of entropy generation was initiated by Bejan [1] by employing the basic laws of thermodynamics, heat transfer process and fluid mechanics. Generally, convective (free, forced, mixed) heat transfer phenomena is related to two types of losses. The first type of loss is caused by the heat transfer through a fixed temperature difference and the second type of loss happens as a consequence of pressure drop in working fluids. These losses are jointly associated with a single property called entropy generation. In other words, an entropy generation is the measure of spices disorder of an energy system. However, several factors like MHD, Joule heating, and diffusion may contribute to irreversibilities which opens the gateway to enhance the thermal efficiency through entropy generation. Ali et al. [2] investigated the entropy generation of chemically reacting Cross nanofluid over a stretched surface with linear radiation and noticed that higher values of Brinkman number and Lorentz force decrease the Bejan number. Sheikholeslami and Ganji [3] employed Koo-Kleinstreuer-Li correlation model to analyze the entropy generation of $\mathrm{CuO}$-water nanofluid in a square enclosure and found that the entropy generation enhances with an increment in nanoparticle volume fraction. Khan et al. [4] numerically scrutinized the stagnation point flow of a Carreau nanofluid with entropy generation and observed that the raising values of diffusive variable augment the entropy generation and Bejan number. Further studies on entropy generation can be found in Refs [5-14].

Fluids which can express the shear stress and shear rate dependent viscosity are classified as non-Newtonian fluids. Biological materials (blood, saliva), chemical materials (polymer fluids, pharmaceutical chemicals), food stuffs (ketchup, yogurt), flow in journal bearings and solar collectors are the examples of the non-Newtonian fluids which find immense applications in biomedical, engineering and technology. The conventional form of Navier-Stokes equations 
is not sufficient to elucidate the flow characteristics of non-Newtonian fluids because of complex rheological properties of non-Newtonian fluids. To overcome this shortcoming, many researchers had proposed several rheological models for non-Newtonian fluids such as Cross fluid, Carreau fluid, Maxwell fluid, Walters B-fluid, Casson fluid and Williamson fluid. Several authors investigated different types of non-Newtonian models in various aspects [15-22]. The hyperbolic tangent fluid is one of the subclasses of non-Newtonian fluids which shows shear thinning characteristics (the viscosity of the fluid is decayed as the shear rate amplifies). Compare to other non-Newtonian fluids, tangent hyperbolic fluid has a lot of advantages like computational easiness, physical robustness, and simplicity. Several experimental results reveal that the tangent hyperbolic fluid model has expressed the exact characteristics of the shear-thinning phenomenon. Moreover, this fluid model accurately expresses the rheological properties of blood flow. The hyperbolic tangent fluid has received notable attention because it is used to analyze the blood flow through a tapered artery and fallopian tube. Gaffar et al. [23] employed a non-similar solution to investigate the heat transfer characteristics of hyperbolic tangent fluid with the convective heating surface and noticed that the hyperbolic tangent fluid temperature enhances with an increment in Weissenberg number. Khan et al.[24] utilized Buongiorno nanofluid model to explore the entropy generation and fluid transport properties of tangent hyperbolic nanofluid over a sheet and found that the skin friction factor increases for increasing Weissenberg number. Nayak et al. [25] examined the importance of activation energy on tangent hyperbolic nanofluid flow over a permeable Riga plate by means of thermophoresis and Brownian motion.

In several engineering and industrial manufacturing process, the difference between the wall and ambient temperatures need not to be small. When the temperature difference is notable, the fluid transport properties are significantly affected. In this case, considering the linear Boussinesq approximation in the fluid transport equation is incapable which leads to reduce the quality of the results. Therefore, it is essential to consider the nonlinear Boussinesq approximation in such cases which certainly contributes to improve the accuracy of the results. It is important to mention that the nonlinear Boussinesq approximation should not be used in low-temperature difference cases. In addition, engineering, geophysical and astrophysical flows are the relevant examples of this nonlinear heat convection (Boussinesq approximation). Vasu et al. [26] discussed entropy generation in the time-dependent mixed convective flow of a nanofluid in the presence of nonlinear Boussinesq approximation and noticed that the uplifting convection parameter increases the nanofluid velocity. Mahanthesh et al. [27] explored the impacts on nonlinear convection and non-linear radiation on Maxwell nanofluid over a three-dimensional extending sheet and observed that the $x$-axis velocity shows an increasing nature for higher values of mixed convection parameter. Waqas et al. [28] used Buongiorno nanofluid to manifest the heat and mass transfer characteristics of magneto-thixotropic nanofluid with an impact of nonlinear convection 
and pointed out that the growing values of the thermophoresis parameter inflate the nanofluid temperature and concentration. Patil et al. [29] scrutinized the hydrogen and oxygen diffusion in nanofluid flow over a roughness cone using triple nonlinear convection and observed that the skin friction factor has an oscillating nature for increasing values of roughness surface parameter. Kameswaran et al. [30] modeled and analyzed the nonlinear convection impact on the boundary layer flow over a permeable plate and noticed that the rising values of the nonlinear temperature parameter tend to decline the thickness of the thermal related boundary layer. The term nanofluid was originated by Choi and Eastman [31] and this fluid is acquired by the dilute suspension of nanometer (1-100 nm)-size solid particles in regular fluids (oil, water etc.). It is observed that the heat transfer efficiency of ordinary fluids is highly enhanced with the inclusion of particles like $\mathrm{ZnO}, \mathrm{Cu}, \mathrm{SiO}_{2}, \mathrm{TiO}_{2}, \mathrm{CuO}$ and $\mathrm{Al}_{2} \mathrm{O}_{3}$ [32-36]. Numerous models have been yielded by researchers to look into the nanofluids, in which the Tiwari and Das model and Buongiorno model are notable. The Tiwari and Das [37] model proposes the behavior of nanofluid with the use of solid volume fraction. In the nanofluid, a nonhomogeneous symmetrical model of four equations of two-components for fluid transport using the influence of slip mechanisms (thermophoresis and Brownian movement) was proposed by Buongiorno [38].

To the best of the authors knowledge, no effort has been made to explore the entropy analysis on hyperbolic tangent nanofluid with nonlinear Boussinesq approximation over a circular cylinder. So, the prime intention of the present model is to exhibit the fluid flow and heat transfer characteristics of hyperbolic tangent nanofluid over a horizontal circular cylinder in existence of nonlinear convection. It is noticed that the mixed convection flow of nonNewtonian nanofluid over a heated horizontal cylinder has notable uses in the geological and industrial process such as geothermal reservoirs, thermal recovery of oil, drag reduction, coating of wires, thrust bearing, underground nuclear waste storage sites and assessment of aquifers. The system of fluid transport equations is solved numerically by using unconditionally stable implicit finite difference Keller box method. Influence of diverse pertinent parameters on the velocity, temperature, concentration and entropy generation are analyzed through the graphs.

\section{Mathematical Formulation}

The schematic view of geometry for the present problem is manifested in Figure 1 which is modeled in a two-dimensional Cartesian coordinate system. The magnetic field strength is assumed to be uniform and is applied parallel to the fluid motion. The coordinates $x$ and $y$ are taken along the circumference of the cylinder and normal to the cylinder, respectively. $a$ is the radius of the cylinder. Changes in density for the buoyancy term is determined by employing nonlinear Boussinesq approximation. The constant temperature $\left(T_{W}\right)$ and 


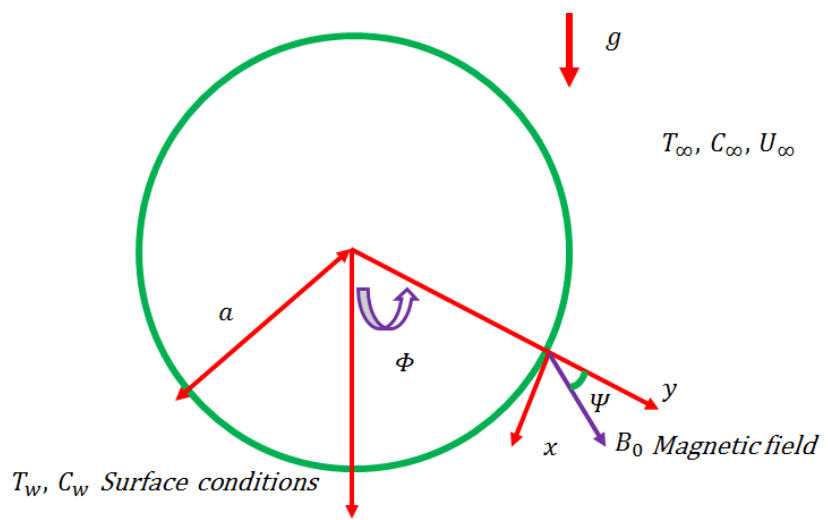

Fig. 1 Physical configuration of the geometry.

concentration $\left(C_{W}\right)$ of the wall is presumed to be greater than the ambient temperature $\left(T_{\infty}\right)$ and ambient concentration $\left(C_{\infty}\right)$, respectively. Buongiorno nanofluid model is employed to express the momentum, energy and concentration equations. In addition, the following assumptions are taken into consideration.

- Laminar, steady, incompressible, mixed convective flow of tangent hyperbolic nanofluid over a circular cylinder is considered.

- The body force is implemented in the momentum equation.

- The induced magnetic field strength is smaller compared to external magnetic field, so it is neglected.

- Tangent hyperbolic nanofluid dissipation and Joule heating are considered in the energy equation.

Based on these assumptions, the flow of tangent hyperbolic nanofluid is governed by the following equations [4, 15, 23, 26 and 27]

$$
\begin{gathered}
\frac{\partial u^{*}}{\partial x}+\frac{\partial v^{*}}{\partial y}=0 \\
u^{*} \frac{\partial u^{*}}{\partial x}+v^{*} \frac{\partial u^{*}}{\partial y}=u_{e}^{*} \frac{d u_{e}^{*}}{d x}+\nu_{f}\left(1-n_{a}\right) \frac{\partial^{2} u^{*}}{\partial y^{2}}+\nu_{f} n_{a} \Gamma \sqrt{2}\left(\frac{\partial u^{*}}{\partial y}\right) \frac{\partial^{2} u^{*}}{\partial y^{2}} \\
+\left[\left(1-C_{\infty}\right) \rho_{f} \beta_{0} g\left(T-T_{\infty}\right)+\left(1-C_{\infty}\right) \rho_{f} \beta_{1} g\left(T-T_{\infty}\right)^{2}\right] \sin \left(\frac{x}{a}\right) \\
\quad-g\left(\rho_{p}-\rho_{f}\right)\left(C-C_{\infty}\right) \sin \left(\frac{x}{a}\right)-\frac{\sigma B_{0}^{2}}{\rho_{f}} \sin ^{2} \Psi\left(u^{*}-u_{e}^{*}\right) \\
u^{*} \frac{\partial T}{\partial x}+v^{*} \frac{\partial T}{\partial y}=\frac{k_{f}}{\left(\rho C_{p}\right)_{f}} \frac{\partial^{2} T}{\partial y^{2}}+\tau\left[D_{B} \frac{\partial C}{\partial y} \frac{\partial T}{\partial y}+\frac{D_{T}}{T_{\infty}}\left(\frac{\partial T}{\partial y}\right)^{2}\right] \\
\quad+\frac{\mu_{f}}{\left(\rho C_{p}\right)_{f}}\left(1-n_{a}\right)\left(\frac{\partial u^{*}}{\partial y}\right)^{2}+\frac{\mu_{f}}{\left(\rho C_{p}\right)_{f}} \frac{n_{a} \Gamma}{\sqrt{2}} \frac{\partial u^{*}}{\partial y}\left(\frac{\partial u^{*}}{\partial y}\right)^{2}
\end{gathered}
$$




$$
\begin{array}{r}
-\frac{1}{\left(\rho C_{p}\right)_{f}} \frac{\partial q_{r}}{\partial y}+\frac{\sigma B_{0}^{2}}{\left(\rho C_{p}\right)_{f}} \sin ^{2} \Psi\left(u^{*}\right)^{2}, \\
u^{*} \frac{\partial C}{\partial x}+v^{*} \frac{\partial C}{\partial y}=D_{B} \frac{\partial^{2} C}{\partial y^{2}}+\frac{D_{T}}{T_{\infty}} \frac{\partial^{2} T}{\partial y^{2}} .
\end{array}
$$

The boundary conditions of this model are as follows

$$
\left.\begin{array}{ll}
u^{*}=0, v^{*}=0, T=T_{W}, C=C_{W} & \text { at } \mathrm{y}=0, \\
u^{*} \rightarrow u_{e}^{*}, \quad T \rightarrow T_{\infty}, \quad C \rightarrow C_{\infty} & \text { as } \mathrm{y} \rightarrow \infty .
\end{array}\right\}
$$

According to Rashad et al. [39], external flow $\left(u_{e}^{*}\right)$ of the fluid transport equation can be expresses as $u_{e}^{*}=\sin \left(\frac{x}{a}\right) U_{\infty}$.

The radiative heat flux [27] is expressed as

$$
q_{r}=-\frac{4}{3} \frac{\sigma_{B}}{k_{a}}\left(\frac{\partial T^{4}}{\partial y}\right)=-\frac{16}{3} \frac{\sigma_{B} T_{\infty}^{3}}{k_{a}}\left(\frac{\partial T}{\partial y}\right),
$$

Suitable non-similarity variables are expressed as follows

$$
\left.\begin{array}{l}
\xi=\frac{x}{a}, \eta=\sqrt{\operatorname{Re}}\left(\frac{y}{a}\right), u=\frac{u^{*}}{U_{\infty}}, v=\sqrt{\operatorname{Re}}\left(\frac{v^{*}}{U_{\infty}}\right), u_{e}(\xi)=\frac{u_{e}^{*}(x)}{U_{\infty}}, \\
T=T_{\infty}+\theta\left(T_{W}-T_{\infty}\right), \quad C=C_{\infty}+\phi\left(C_{W}-C_{\infty}\right) .
\end{array}\right\}
$$

where $\operatorname{Re}=\frac{a U_{\infty}}{\nu_{f}}$.

By implementing the above variables, Eqns. (1)-(4) are transformed as

$$
\begin{aligned}
& \frac{\partial u}{\partial \xi}+\frac{\partial v}{\partial \eta}=0 \\
& u \frac{\partial u}{\partial \xi}+v \frac{\partial u}{\partial \eta}=u_{e} \frac{d u_{e}}{d \xi}+\left(1-n_{a}\right) \frac{\partial^{2} u}{\partial \eta^{2}}+n_{a} W e \frac{\partial u}{\partial \eta} \frac{\partial^{2} u}{\partial \eta^{2}} \\
& -M_{a} \sin ^{2} \Psi\left(u-u_{e}\right)+\lambda_{c}\left(\theta+N_{c} \theta^{2}-N_{r} \phi\right) \sin \xi, \\
& u \frac{\partial \theta}{\partial \xi}+v \frac{\partial \theta}{\partial \eta}=\frac{1}{\operatorname{Pr}}\left(1+\frac{4}{3} R_{a}\right) \frac{\partial^{2} \theta}{\partial \eta^{2}}+N_{B} \frac{\partial \theta}{\partial \eta} \frac{\partial \phi}{\partial \eta}+N_{T}\left(\frac{\partial \theta}{\partial \eta}\right)^{2} \\
& +M_{a} E_{c} \sin ^{2} \Psi(u)^{2}+E_{c}\left(1-n_{a}\right)\left(\frac{\partial u}{\partial \eta}\right)^{2}+E_{c} \frac{n_{a} W e}{2} \frac{\partial u}{\partial \eta}\left(\frac{\partial u}{\partial \eta}\right)^{2} \\
& u \frac{\partial \phi}{\partial \xi}+v \frac{\partial \phi}{\partial \eta}=\frac{1}{S c}\left[\frac{\partial^{2} \phi}{\partial \eta^{2}}+\frac{N_{T}}{N_{B}} \frac{\partial^{2} \theta}{\partial \eta^{2}}\right] .
\end{aligned}
$$

with the corresponding boundary conditions

$$
\left.\begin{array}{l}
u=0, v=0, \theta=1, \phi=1 \text { at } \eta=0, \\
u \rightarrow u_{e}, \quad \theta \rightarrow 0, \quad \phi \rightarrow 0 \quad \text { as } \eta \rightarrow \infty .
\end{array}\right\}
$$

where $\lambda_{c}=\frac{\left(1-C_{\infty}\right) g \beta_{0}\left(T_{W}-T_{\infty}\right) a}{U_{\infty}^{2}}, W e=\frac{\Gamma \sqrt{\operatorname{Re}} \sqrt{2} U_{\infty}}{a}, \operatorname{Pr}=\frac{\nu_{f}}{\alpha^{*}}, M_{a}=\frac{\sigma B_{0}^{2} a}{U_{\infty} \rho_{f}}$, $N_{c}=\frac{\beta_{1}\left(T_{W}-T_{\infty}\right)}{\beta_{0}}, \quad N_{r}=\frac{\left(\rho_{p}-\rho_{f}\right)\left(C_{W}-C_{\infty}\right)}{\left(1-C_{\infty}\right) \rho_{f} \beta_{0}\left(T_{W}-T_{\infty}\right)}, \quad E_{c}=\frac{U_{\infty}^{2}}{\left(C_{p}\right)_{f}\left(T_{W}-T_{\infty}\right)}, N_{B}=$ 
$\frac{\tau D_{B}\left(C_{W}-C_{\infty}\right)}{\nu_{f}}, R_{a}=\frac{4 \sigma_{B} T_{\infty}^{3}}{k_{a} k_{f}}, N_{T}=\frac{\tau D_{B}\left(T_{W}-T_{\infty}\right)}{\nu_{f} T_{\infty}}$ and $S c=\frac{\nu_{f}}{D_{B}}$.

To expresses the flow equations with the boundary conditions in dimensionless form, $\psi=\xi f(\xi, \eta), \theta=\theta(\xi, \eta), \phi=\phi(\xi, \eta)$ are assumed according to Rashad et al. [39], where $\psi$ is the stream function which is defined as $u=\frac{\partial(\psi(\xi, \eta))}{\partial \eta} \& v=-\frac{\partial(\psi(\xi, \eta))}{\partial \xi}$.

$$
\begin{aligned}
& \left(1-n_{a}\right) f^{\prime \prime \prime}+n_{a} W e f^{\prime \prime \prime} f^{\prime \prime}+f f^{\prime \prime}-\left(f^{\prime}\right)^{2}+\frac{\sin \xi \cos \xi}{\xi}-M_{a} \sin ^{2} \Psi \\
& \left(1-\frac{\sin \xi}{\xi}\right)+\lambda_{c} \frac{\sin \xi}{\xi}\left(\theta+N_{c} \theta^{2}-N_{r} \phi\right)=\xi\left[f^{\prime} \frac{\partial f^{\prime}}{\partial \xi}-f^{\prime \prime} \frac{\partial f}{\partial \xi}\right], \\
& \frac{1}{\operatorname{Pr}}\left[1+\frac{4}{3} R_{a}\right] \theta^{\prime \prime}+f \theta^{\prime}+N_{B} \theta^{\prime} \phi^{\prime}+N_{T}\left(\theta^{\prime}\right)^{2}+E_{c} \xi^{2} M \sin ^{2} \Psi\left(f^{\prime}\right)^{2} \\
& +E_{c} \xi^{2}\left(1-n_{a}\right)\left(f^{\prime \prime}\right)^{2}+E_{c} \xi^{3} n_{a} \frac{W e}{2}\left(f^{\prime \prime}\right)^{3}=\xi\left[f^{\prime} \frac{\partial \theta}{\partial \xi}-\theta^{\prime} \frac{\partial f}{\partial \xi}\right], \\
& \frac{1}{S c}\left[\phi^{\prime \prime}+\frac{N_{T}}{N_{B}} \theta^{\prime \prime}\right]+f \phi^{\prime}=\xi\left[f^{\prime} \frac{\partial \phi}{\partial \xi}-\phi^{\prime} \frac{\partial f}{\partial \xi}\right] .
\end{aligned}
$$

along with the transformed boundary conditions

$$
\left.\begin{array}{ll}
f=f^{\prime}=0, \quad \theta=\phi=1 & \text { at } \eta=0 \\
f^{\prime} \rightarrow 1, \quad \theta \rightarrow 0, \quad \phi \rightarrow 0 & \text { as } \eta \rightarrow \infty .
\end{array}\right\}
$$

Table 1 Comparison result of $N u^{*}$ for various values of $\xi$ with $\operatorname{Pr}=0.71, R_{a}=0, M_{a}=0.5$, $S c=0.6, W e=u_{e}=N_{r}=E_{c}=n_{a}=N_{B}=N_{T}=N_{c}=0, \Psi=90^{\circ}$

\begin{tabular}{|c|c|c|c|c|}
\hline \multirow{2}{*}{$\xi$} & \multicolumn{4}{|c|}{$N u^{*}(G r)^{-1 / 4}=-\theta^{\prime}(\xi, 0)$} \\
\cline { 2 - 5 } & Merkin[40] & Yih[41] & Prasad et al.[22] & Present \\
\hline 0.0 & 0.4212 & 0.4214 & 0.4211 & 0.4211 \\
\hline 0.2 & 0.4204 & 0.4207 & 0.4206 & 0.4206 \\
\hline 0.4 & 0.4182 & 0.4184 & 0.4185 & 0.4185 \\
\hline 0.6 & 0.4145 & 0.4147 & 0.4146 & 0.4146 \\
\hline 0.8 & 0.4093 & 0.4096 & 0.4095 & 0.4095 \\
\hline 1.0 & 0.4025 & 0.4030 & 0.4027 & 0.4027 \\
\hline
\end{tabular}

At the wall, the dimensionless forms of skin friction factor $\left(C_{f}^{*}\right)$, heat transfer rate $\left(N u^{*}\right)$ and mass transfer rate $\left(S h^{*}\right)$ are defined as

$$
\left.\begin{array}{l}
C_{f}^{*}\left(G r^{-3 / 4}\right)=\left(1-n_{a}\right) \xi f^{\prime \prime}(\xi, 0)+\frac{n_{a}}{2} W e \xi\left(f^{\prime \prime}(\xi, 0)\right)^{2}, \\
N u^{*}\left(G r^{-1 / 4}\right)=-\left(1+\frac{4}{3} R_{a}\right) \theta^{\prime}(\xi, 0), \\
S h^{*}\left(G r^{-1 / 4}\right)=-\phi^{\prime}(\xi, 0) .
\end{array}\right\}
$$




\subsection{Entropy generation}

The dimensional form of entropy generation for nanofluid can be written as follows [22 and 24]:

$$
\begin{gathered}
S_{G}=\underbrace{\frac{k_{f}}{T_{\infty}^{2}}\left(1+\frac{16 \sigma_{B} T_{\infty}^{3}}{3 k_{f} k_{a}}\right)\left(\frac{\partial T}{\partial y}\right)^{2}}_{\text {Thermal irreversibility }}+\underbrace{\frac{R D}{C_{\infty}}\left(\frac{\partial C}{\partial y}\right)^{2}+\frac{R D}{T_{\infty}}\left(\frac{\partial T}{\partial y} \frac{\partial C}{\partial y}\right)}_{\text {Mass irreversibility }} \\
+\underbrace{\frac{\mu_{f}}{T_{\infty}}\left[\left(1-n_{a}\right)\left(\frac{\partial u^{*}}{\partial y}\right)^{2}+\frac{n_{a} \Gamma}{\sqrt{2}} \frac{\partial u^{*}}{\partial y}\left(\frac{\partial u^{*}}{\partial y}\right)^{2}\right]}_{\text {Tangent hyperbolic nanofluid friction irreversibility }}+\underbrace{\frac{\sigma}{T_{\infty}} B_{0}^{2} \sin ^{2} \Psi\left(u^{*}\right)^{2}}_{\text {Joule heating irreversibility }},
\end{gathered}
$$

where, $D$ is the mass diffusivity and $R$ is the constant.

Using Eqn. (7) in Eqn. (18), the transformed expression for entropy generation is

$$
\begin{aligned}
N_{G}=\alpha_{T}(1+ & \left.\frac{4}{3} R_{a}\right)\left(\frac{\partial \theta}{\partial \eta}\right)^{2}+B r\left(1-n_{a}\right)\left(\frac{\partial u}{\partial \eta}\right)^{2}+B r \frac{n_{a} W e}{2} \frac{\partial u}{\partial \eta}\left(\frac{\partial u}{\partial \eta}\right)^{2} \\
& +M_{a} B r \sin ^{2} \Psi(u)^{2}+L_{T}\left(\frac{\alpha_{C}}{\alpha_{T}}\right)\left(\frac{\partial \phi}{\partial \eta}\right)^{2}+L_{T} \frac{\partial \theta}{\partial \eta} \frac{\partial \phi}{\partial \eta}
\end{aligned}
$$

where $N_{G}=\frac{S_{G} T_{\infty} a}{k_{f}\left(T_{W}-T_{\infty}\right) \operatorname{Re}}, \alpha_{T}=\frac{T_{W}-T_{\infty}}{T_{\infty}}, \alpha_{C}=\frac{C_{W}-C_{\infty}}{C_{\infty}}, L_{T}=\frac{R D\left(C_{W}-C_{\infty}\right)}{k_{f}}$ and $B r=\frac{\mu_{f} U_{\infty}^{2}}{k_{f}\left(T_{W}-T_{\infty}\right)}$.

Employing $\psi, \theta$ and $\phi$ function for dimensionless form of total entropy generation

$$
\begin{aligned}
N_{G}=\alpha_{T}(1+ & \left.\frac{4}{3} R_{a}\right)\left(\theta^{\prime}\right)^{2}+B r \xi^{2}\left(1-n_{a}\right)\left(f^{\prime \prime}\right)^{2}+B r \xi^{3} \frac{n_{a} W e}{2}\left(f^{\prime \prime}\right)^{3} \\
& +M_{a} B r \xi^{2} \sin ^{2} \Psi\left(f^{\prime}\right)^{2}+L_{T}\left(\frac{\alpha_{C}}{\alpha_{T}}\right)\left(\phi^{\prime}\right)^{2}+L_{T} \theta^{\prime} \phi^{\prime} .
\end{aligned}
$$

Further, Bejan number is expressed as the ratio between entropy generation due to heat and mass transfer and total entropy generation

$$
\left.\begin{array}{rl}
B e= & \frac{\alpha_{T}\left(1+\frac{4}{3} R_{a}\right)\left(\theta^{\prime}\right)^{2}+L_{T}\left(\frac{\alpha_{C}}{\alpha_{T}}\right)\left(\phi^{\prime}\right)^{2}+L_{T} \theta^{\prime} \phi^{\prime}}{\alpha_{T}\left(1+\frac{4}{3} R_{a}\right)\left(\theta^{\prime}\right)^{2}+B r \xi^{2}\left(1-n_{a}\right)\left(f^{\prime \prime}\right)^{2}} \\
& +B r \xi^{3} \frac{n_{a} W e}{2}\left(f^{\prime \prime}\right)^{3}+M_{a} B r \xi^{2} \sin ^{2} \Psi\left(f^{\prime}\right)^{2} \\
& +L_{T}\left(\frac{\alpha_{C}}{\alpha_{T}}\right)\left(\phi^{\prime}\right)^{2}+L_{T} \theta^{\prime} \phi^{\prime}
\end{array}\right\}=N_{G}
$$


Table 2 Impacts of $n_{a}$ and $N_{c}$ on local skin friction coefficient $\left(C_{f}^{*}\right)$, dimensionless local rate of heat transfer $\left(N u^{*}\right)$ and dimensionless local rate of mass transfer for various values of $\xi$.

\begin{tabular}{|c|c|c|c|c|c|c|c|c|c|}
\hline \multirow{2}{*}{$\begin{array}{c}\text { Physical } \\
\text { Parameters }\end{array}$} & \multirow[t]{2}{*}{ Values } & \multirow{2}{*}{$\begin{array}{c}\text { Physical } \\
\text { Quantities }\end{array}$} & \multicolumn{6}{|c|}{$\xi$} & \multirow{2}{*}{$\begin{array}{c}\text { CPU } \\
\text { time(Sec.) }\end{array}$} \\
\hline & & & 0 & 0.2 & 0.4 & 0.6 & 0.8 & 1 & \\
\hline \multirow{12}{*}{$n_{a}$} & \multirow{3}{*}{0.1} & $C_{f}^{*}$ & 0 & 0.3803 & 0.7283 & 1.0230 & 1.2482 & 1.3938 & \multirow{3}{*}{10.848590} \\
\hline & & $N u^{*}$ & 1.1063 & 1.0954 & 1.0738 & 1.0421 & 1.0010 & 0.9514 & \\
\hline & & $S h^{*}$ & 0.8271 & 0.8201 & 0.8061 & 0.7853 & 0.7578 & 0.7239 & \\
\hline & \multirow{3}{*}{0.3} & $C_{f}^{*}$ & 0 & 0.3672 & 0.7013 & 0.9810 & 1.1906 & 1.3208 & \multirow{3}{*}{11.069575} \\
\hline & & $N u^{*}$ & 1.1097 & 1.0992 & 1.0783 & 1.0475 & 1.0075 & 0.9589 & \\
\hline & & $S h^{*}$ & 0.8291 & 0.8225 & 0.8093 & 0.7897 & 0.7636 & 0.7314 & \\
\hline & \multirow{3}{*}{0.5} & $C_{f}^{*}$ & 0 & 0.3542 & 0.6744 & 0.9393 & 1.1334 & 1.2482 & \multirow{3}{*}{11.046161} \\
\hline & & $N u^{*}$ & 1.1132 & 1.1031 & 1.0829 & 1.0530 & 1.0139 & 0.9664 & \\
\hline & & $S h^{*}$ & 0.8314 & 0.8252 & 0.8127 & 0.7940 & 0.7693 & 0.7387 & \\
\hline & \multirow{3}{*}{0.8} & $C_{f}^{*}$ & 0 & 0.3348 & 0.6346 & 0.8777 & 1.0487 & 1.1401 & \multirow{3}{*}{11.207880} \\
\hline & & $N u^{*}$ & 1.1188 & 1.1092 & 1.0899 & 1.0612 & 1.0236 & 0.9777 & \\
\hline & & $S h^{*}$ & 0.8354 & 0.8296 & 0.8180 & 0.8008 & 0.7779 & 0.7495 & \\
\hline \multirow{12}{*}{$N_{c}$} & \multirow{3}{*}{0.1} & $C_{f}^{*}$ & 0 & 0.3672 & 0.7013 & 0.9810 & 1.1906 & 1.3208 & \multirow{3}{*}{10.820276} \\
\hline & & $N u^{*}$ & 1.1097 & 1.0992 & 1.0783 & 1.0475 & 1.0075 & 0.9589 & \\
\hline & & $S h^{*}$ & 0.8291 & 0.8225 & 0.8093 & 0.7897 & 0.7636 & 0.7314 & \\
\hline & \multirow{3}{*}{0.5} & $C_{f}^{*}$ & 0 & 0.4042 & 0.7741 & 1.0876 & 1.3280 & 1.4855 & \multirow{3}{*}{11.010522} \\
\hline & & $N u^{*}$ & 1.1257 & 1.1154 & 1.0949 & 1.0647 & 1.0254 & 0.9779 & \\
\hline & & $S h^{*}$ & 0.8469 & 0.8405 & 0.8279 & 0.8090 & 0.7841 & 0.7533 & \\
\hline & \multirow{3}{*}{1.0} & $C_{f}^{*}$ & 0 & 0.4499 & 0.8639 & 1.2189 & 1.4973 & 1.6879 & \multirow{3}{*}{11.057783} \\
\hline & & $N u^{*}$ & 1.1445 & 1.1343 & 1.1142 & 1.0845 & 1.0459 & 0.9994 & \\
\hline & & $S h^{*}$ & 0.8674 & 0.8613 & 0.8492 & 0.8312 & 0.8075 & 0.7782 & \\
\hline & \multirow{3}{*}{1.5} & $C_{f}^{*}$ & 0 & 0.4950 & 0.9526 & 1.3485 & 1.6641 & 1.8871 & \multirow{3}{*}{10.904997} \\
\hline & & $N u^{*}$ & 1.1620 & 1.1520 & 1.1321 & 1.1028 & 1.0647 & 1.0188 & \\
\hline & & $S h^{*}$ & 0.8864 & 0.8805 & 0.8689 & 0.8516 & 0.8288 & 0.8006 & \\
\hline
\end{tabular}

\section{Numerical method and code validation}

The dimensionless equations under the suitable conditions are solved numerically using unconditionally stable implicit finite difference scheme which is known as the Keller box method (KBM). The present numerical scheme is second-order accurate for the boundary layer problem which is represented using parabolic type partial differential equations(PDEs). The procedure to obtain the solution by using the Keller box method is given below

- The $n^{\text {th }}$ order dimensionless equations are transformed into $n$ first order dimensionless equations.

- The transformed $n$ first order equations are discretized by employing central differences approach.

- The algebraic equations are linearized by means of Newton's method.

- The results are obtained by using the block matrix system (block tridiagonal elimination technique).

Step 1:

We have considered a new set of variables $u(\xi, \eta), v(\xi, \eta), s(\xi, \eta), t(\xi, \eta), g(\xi, \eta), p(\xi, \eta)$ 
to transform the $n^{\text {th }}$ order dimensionless equations into the first order dimensionless equations, which depends on $\xi$ and $\eta$.

$$
\begin{aligned}
& f=f, f^{\prime}=u, u^{\prime}=v, \theta=s, s^{\prime}=t, \phi=g, g^{\prime}=p \\
& \left(1-n_{a}\right) \xi v^{\prime}+n_{a} W e \xi v^{\prime} v+f v-u^{2}+\frac{\sin \xi \cos \xi}{\xi}-M_{a} \sin ^{2} \Psi \\
& \left(u-\frac{\sin \xi}{\xi}\right)+\lambda_{c} \frac{\sin \xi}{\xi}\left(s+N_{c} s^{2}-N_{r} g\right)=\xi\left[u \frac{\partial u}{\partial \xi}-v \frac{\partial f}{\partial \xi}\right], \\
& \frac{1}{\operatorname{Pr}}\left[1+\frac{4}{3} R_{a}\right] t^{\prime}+N_{T} t^{2}+N_{B} t p+M_{a} E_{c} \xi^{2} \sin ^{2} \Psi(u)^{2}+f t \\
& \quad E_{c}\left(1-n_{a}\right) \xi^{2} v^{2}+E_{c} \frac{n_{a} W e}{2} \xi^{3} v^{3}=\xi\left[u \frac{\partial s}{\partial \xi}-t \frac{\partial f}{\partial \xi}\right], \\
& \frac{1}{S c}\left[p^{\prime}+\frac{N_{T}}{N_{B}} t^{\prime}\right]+f p=\xi\left[u \frac{\partial g}{\partial \xi}-p \frac{\partial f}{\partial \xi}\right] .
\end{aligned}
$$

with the boundary conditions

$$
\begin{array}{ll}
\eta=0: & u=0, \quad f=0, \quad s=1, \quad g=1 \\
\eta \rightarrow \infty: & u \rightarrow 1, \quad s=0, \quad g=0
\end{array}
$$

\section{Step 2:}

The net point of $x$ and $y$ plane is expressed as

$$
\begin{aligned}
& \xi^{0}=0, \quad \xi^{i}=\xi^{i-1}+k_{i}, i=1,2,3 \ldots I, \\
& \eta_{0}=0, \quad \eta_{j}=\eta_{j-1}+h_{j}, \quad j=1,2,3 \ldots J .
\end{aligned}
$$

where $k_{i}$ is $\Delta \xi$ spacing in the $i^{t h}$ node and $h_{j}$ is $\Delta \eta$ spacing in $j^{\text {th }}$ node.

$$
\begin{aligned}
& \left(\frac{\partial()}{\partial \xi}\right)_{j-\frac{1}{2}}^{i-\frac{1}{2}}=\frac{()_{j-\frac{1}{2}}^{i}-()_{j-\frac{1}{2}}^{i-1}}{k_{i}},\left(\frac{\partial()}{\partial \eta}\right)_{j-\frac{1}{2}}^{i-\frac{1}{2}}=\frac{()_{j}^{i-\frac{1}{2}}-()_{j-1}^{i-\frac{1}{2}}}{h_{j}} \\
& ()_{j}^{i-\frac{1}{2}}=\frac{()_{j}^{i-1}-()_{j}^{i}}{2},()_{j-\frac{1}{2}}^{i}=\frac{()_{j-1}^{i}-()_{j}^{i}}{2}
\end{aligned}
$$

The midpoint $\left(\xi^{i}, \eta_{j-\frac{1}{2}}\right)$ is considered between the segments $\left(\xi^{i}, \eta_{j-1}\right)\left(\xi^{i}, \eta_{j}\right)$ by using central difference approximation

$$
\begin{gathered}
f^{\prime}=u \Rightarrow u_{j-\frac{1}{2}}^{i}=\frac{u_{j}^{i}+u_{j-1}^{i}}{2}=\frac{\left(f_{j}^{i}-f_{j-1}^{i}\right)}{h_{j}}, \\
u^{\prime}=v \Rightarrow v_{j-\frac{1}{2}}^{i}=\frac{v_{j}^{i}+v_{j-1}^{i}}{2}=\frac{\left(u_{j}^{i}-u_{j-1}^{i}\right)}{h_{j}}
\end{gathered}
$$




$$
\begin{gathered}
s^{\prime}=t \Rightarrow t_{j-\frac{1}{2}}^{i}=\frac{t_{j}^{i}+t_{j-1}^{i}}{2}=\frac{\left(s_{j}^{i}-s_{j-1}^{i}\right)}{h_{j}} \\
g^{\prime}=p \Rightarrow p_{j-\frac{1}{2}}^{i}=\frac{p_{j}^{i}+p_{j-1}^{i}}{2}=\frac{\left(g_{j}^{i}-g_{j-1}^{i}\right)}{h_{j}},
\end{gathered}
$$

First order PDEs Eqns. (23)-(25) are approximated by centering the rectangle points $\left(P_{1}, P_{2}, P_{3}\right.$, and $\left.P_{4}\right)$ at $\left(\xi^{i-\frac{1}{2}}, \eta_{j-\frac{1}{2}}\right)$

$$
\begin{aligned}
& \left(\frac{v_{j}^{i}-v_{j-1}^{i}}{h_{j}}\right)\left(1-n_{a}\right) \xi+n_{a} W e \xi v_{j-1 / 2}^{i}\left(\frac{v_{j}^{i}-v_{j-1}^{i}}{h_{j}}\right) \\
& +(1+\alpha)\left(f_{j-1 / 2}^{i} v_{j-1 / 2}^{i}\right)-(1+\alpha)\left(u_{j-1 / 2}^{i}\right)^{2}-\left(M_{a} \sin ^{2} \Psi\right) u_{j-1 / 2}^{i} \\
& +M_{a} \sin ^{2} \Psi 2 B+2 H+\lambda_{c} B\left(s_{j-1 / 2}^{i}+N_{c}\left(s_{j-1 / 2}^{i}\right)^{2}-N_{r} g_{j-1 / 2}^{i}\right) \\
& +\alpha v_{j-1 / 2}^{i-1} f_{j-1 / 2}^{i}-\alpha f_{j-1 / 2}^{i-1} v_{j-1 / 2}^{i} \\
& =-\left[\begin{array}{l}
\left(\frac{v_{j}^{i}-v_{j-1}^{i}}{h_{j}}\right)\left(1-n_{a}\right) \xi+n_{a} W e \xi v_{j-1 / 2}^{i}\left(\frac{v_{j}^{i}-v_{j-1}^{i}}{h_{j}}\right) \\
+(1-\alpha)\left(f_{j-1 / 2}^{i-1} v_{j-1 / 2}^{i-1}\right)+(\alpha-1)\left(u_{j-1 / 2}^{i-1}\right)^{2} \\
+\lambda_{c} B\left(s_{j-1 / 2}^{i}+N_{c}\left(s_{j-1 / 2}^{i}\right)^{2}-N_{r} g_{j-1 / 2}^{i}\right)^{i} \\
-\left(M_{a} \sin ^{2} \Psi\right) u_{j-1 / 2}^{i-1}
\end{array}\right] \\
& \left.\frac{1}{\operatorname{Pr}}\left[1+\frac{4}{3} R_{a}\right]\left(\frac{t_{j}^{i}-t_{j-1}^{i}}{h_{j}}\right)+N_{B}\left(t_{j-1 / 2}^{i} p_{j-1 / 2}^{i}\right)+N_{T}\left(t_{j-1 / 2}^{i}\right)^{2}\right) \\
& +M_{a} E_{c} \sin ^{2} \Psi \xi^{2}\left(u_{j-1 / 2}^{i}\right)^{2}+E_{c}\left(1-n_{a}\right) \xi^{2}\left(v_{j-1 / 2}^{i}\right)^{2} \\
& +E_{c} \frac{n_{a} W e}{2} \xi^{3}\left(v_{j-1 / 2}^{i}\right)^{3}-\alpha u_{j-1 / 2}^{i-1} s_{j-1 / 2}^{i}-\alpha f_{j-1 / 2}^{i-1} t_{j-1 / 2}^{i} \\
& +\alpha t_{j-1 / 2}^{i-1} f_{j-1 / 2}^{i}+(1+\alpha)\left(f_{j-1 / 2}^{i} t_{j-1 / 2}^{i}\right) \\
& -\alpha\left(u_{j-1 / 2}^{i} s_{j-1 / 2}^{i}\right)+\alpha s_{j-1 / 2}^{i-1} u_{j-1 / 2}^{i} \\
& =-\left[\begin{array}{l}
\frac{1}{\operatorname{Pr}}\left[1+\frac{4}{3} R_{a}\right]\left(\frac{t_{j}^{i}-t_{j-1}^{i}}{h_{j}}\right)+N_{B}\left(t_{j-1 / 2}^{i} p_{j-1 / 2}^{i}\right) \\
+M_{a} E_{c} \sin ^{2} \Psi \xi^{2}\left(u_{j-1 / 2}^{i}\right)^{2}+E_{c}\left(1-n_{a}\right) \xi^{2}\left(v_{j-1 / 2}^{i}\right)^{2} \\
+E_{c} \frac{n_{a} W e}{2} \xi^{3}\left(v_{j-1 / 2}^{i}\right)^{3}+N_{T}\left(t_{j-1 / 2}^{i}\right)^{2} \\
+(1-\alpha)\left(f_{j-1 / 2}^{i-1} t_{j-1 / 2}^{i-1}\right)+\alpha\left(u_{j-1 / 2}^{i-1} s_{j-1 / 2}^{i-1}\right)
\end{array}\right] \\
& \frac{1}{S c}\left(\frac{p_{j}^{i}-p_{j-1}^{i}}{h_{j}}\right)+\frac{1}{S c}\left(\frac{N_{T}}{N_{B}}\right)\left(\frac{t_{j}^{i}-t_{j-1}^{i}}{h_{j}}\right) \\
& +(1+\alpha)\left(f_{j-1 / 2}^{i} p_{j-1 / 2}^{i}\right)-\alpha\left(u_{j-1 / 2}^{i} g_{j-1 / 2}^{i}\right)+\alpha g_{j-1 / 2}^{i-1} u_{j-1 / 2}^{i} \\
& -\alpha u_{j-1 / 2}^{i-1} g_{j-1 / 2}^{i}-\alpha f_{j-1 / 2}^{i-1} p_{j-1 / 2}^{i}+\alpha p_{j-1 / 2}^{i-1} f_{j-1 / 2}^{i} \\
& =-\left[\begin{array}{l}
\frac{1}{S c}\left(\frac{p_{j}^{i}-p_{j-1}^{i}}{h_{j}}\right)+\frac{1}{S c}\left(\frac{N_{T}}{N_{B}}\right)\left(\frac{t_{j}^{i}-t_{j-1}^{i}}{h_{j}}\right) \\
+(1-\alpha)\left(f_{j-1 / 2}^{i-1} p_{j-1 / 2}^{i-1}\right)+\alpha\left(u_{j-1 / 2}^{i-1} g_{j-1 / 2}^{i-1}\right)
\end{array}\right]
\end{aligned}
$$


where $\alpha=\frac{\xi^{n-1 / 2}}{k_{n}}, B=\frac{\sin \left(\xi^{n-1 / 2}\right)}{\xi^{n-1 / 2}}$ and $H=\frac{\sin \left(\xi^{n-1 / 2}\right) \cos \left(\xi^{n-1 / 2}\right)}{\xi^{n-1 / 2}}$.

subject to the boundary conditions

$$
f_{0}^{i}=u_{0}^{i}=0, s_{0}^{i}=1, g_{0}^{i}=1, u_{J}^{i} \rightarrow 1, s_{J}^{i}=0, g_{J}^{i}=0
$$

\section{Step 3:}

The unknowns $\left(f_{j}^{i}, u_{j}^{i}, v_{j}^{i}, g_{j}^{i}, p_{j}^{i}, s_{j}^{i}, t_{j}^{i}\right)$ are calculated by using the known values of $f_{j}^{i-1}, u_{j}^{i-1}, v_{j}^{i-1}, g_{j}^{i-1}, p_{j}^{i-1}, s_{j}^{i-1}, t_{j}^{i-1}$ where $0 \leq \mathrm{j} \leq \mathrm{J}$.

The unknowns are assumed as $\left(f_{j}^{i}, u_{j}^{i}, v_{j}^{i}, g_{j}^{i}, p_{j}^{i}, s_{j}^{i}, t_{j}^{i}\right) \equiv\left(f_{j}, u_{j}, v_{j}, g_{j}, p_{j}, s_{j}, t_{j}\right)$.

The set of central difference equations are expressed as

$$
\begin{aligned}
& \frac{u_{j}+u_{j-1}}{2}=\frac{f_{j}-f_{j-1}}{h_{j}}, \\
& \frac{v_{j}+v_{j-1}}{2}=\frac{u_{j}-u_{j-1}}{h_{j}}, \\
& \frac{t_{j}+t_{j-1}}{2}=\frac{s_{j}-s_{j-1}}{h_{j}}, \\
& \frac{p_{j}+p_{j-1}}{2}=\frac{g_{j}-g_{j-1}}{h_{j}},
\end{aligned}
$$

$$
\left.\begin{array}{l}
\left(v_{j}-v_{j-1}\right)\left(1-n_{a}\right) \xi+n_{a} W e \xi \frac{\left(v_{j}+v_{j-1}\right)}{2}\left(v_{j}-v_{j-1}\right)+2 h_{j} H \\
+\frac{(1+\alpha) h_{j}}{4}\left[\left(f_{j}+f_{j-1}\right)\left(v_{j}+v_{j-1}\right)\right]-\frac{h_{j}}{4}(1+\alpha)\left(u_{j}+u_{j-1}\right)^{2} \\
-\frac{h_{j}}{2}\left(M_{a} \sin ^{2} \Psi\right)\left(u_{j}+u_{j-1}\right)+h_{j} M_{a} \sin ^{2} \Psi 2 B \\
-\frac{\alpha h_{j}}{2} f_{j-1 / 2}^{i-1}\left(v_{j}+v_{j-1}\right)+\frac{\alpha h_{j}}{2} v_{j-1 / 2}^{i-1}\left(f_{j}+f_{j-1}\right) \\
+\frac{\lambda_{c} B h_{j}}{2}\left[\left(s_{j}+s_{j-1}\right)+N_{c} \frac{\left(s_{j}+s_{j-1}\right)^{2}}{2}-N_{r}\left(g_{j}+g_{j-1}\right)\right]=\left[E_{1}\right]_{j-1 / 2}^{i-1}
\end{array}\right\}
$$


where,

$$
\begin{aligned}
& {\left[E_{1}\right]_{j-1 / 2}^{i-1}=-h_{j}\left[\begin{array}{l}
\left(\frac{v_{j}-v_{j-1}}{h_{j}}\right)\left(1-n_{a}\right) \xi+n_{a} W e \xi v_{j-1 / 2}\left(\frac{v_{j}-v_{j-1}}{h_{j}}\right) \\
+(1-\alpha)\left(f_{j-1 / 2} v_{j-1 / 2}\right)+(\alpha-1)\left(u_{j-1 / 2}\right)^{2} \\
+\lambda_{c} B\left(s_{j-1 / 2}+N_{c}\left(s_{j-1 / 2}\right)^{2}-N_{r} g_{j-1 / 2}\right) \\
-\left(M_{a} \sin ^{2} \Psi\right) u_{j-1 / 2}
\end{array}\right]} \\
& {\left[E_{2}\right]_{j-1 / 2}^{i-1}=-h_{j}\left[\begin{array}{l}
\frac{1}{\operatorname{Pr}}\left[1+\frac{4}{3} R_{a}\right]\left(\frac{t_{j}-t_{j-1}}{h_{j}}\right)+N_{B}\left(t_{j-1 / 2} p_{j-1 / 2}\right) \\
+M_{a} E_{c} \sin ^{2} \Psi \xi^{2}\left(u_{j-1 / 2}\right)^{2}+E_{c}\left(1-n_{a}\right) \xi^{2}\left(v_{j-1 / 2}\right)^{2} \\
+E_{c} \frac{n_{a} W e}{2} \xi^{3}\left(v_{j-1 / 2}\right)^{3}+N_{T}\left(t_{j-1 / 2}\right)^{2} \\
+(1-\alpha)\left(f_{j-1 / 2} t_{j-1 / 2}\right)+\alpha\left(u_{j-1 / 2} s_{j-1 / 2}\right)
\end{array}\right](45)} \\
& {\left[E_{3}\right]_{j-1 / 2}^{i-1}=-h_{j}\left[\begin{array}{l}
\frac{1}{S c}\left(\frac{p_{j}-p_{j-1}}{h_{j}}\right)+\frac{1}{S c}\left(\frac{N_{T}}{N_{B}}\right)\left(\frac{t_{j}-t_{j-1}}{h_{j}}\right) \\
+(1-\alpha)\left(f_{j-1 / 2} p_{j-1 / 2}\right)+\alpha\left(u_{j-1 / 2} g_{j-1 / 2}\right)
\end{array}\right]}
\end{aligned}
$$

To linearize the nonlinear system of equations using Newtons method, the following iterates are introduced

$$
\begin{aligned}
& \omega f_{j}^{(n)}=f_{j}^{(n+1)}-f_{j}^{(n)}, \\
& \omega u_{j}^{(n)}=u_{j}^{(n+1)}-u_{j}^{(n)}, \\
& \omega v_{j}^{(n)}=v_{j}^{(n+1)}-v_{j}^{(n)}, \\
& \omega s_{j}^{(n)}=s_{j}^{(n+1)}-s_{j}^{(n)}, \\
& \omega t_{j}^{(n)}=t_{j}^{(n+1)}-t_{j}^{(n)}, \\
& \omega g_{j}^{(n)}=g_{j}^{(n+1)}-g_{j}^{(n)}, \\
& \omega p_{j}^{(n)}=p_{j}^{(n+1)}-p_{j}^{(n)}
\end{aligned}
$$

Implementing the above mentioned expressions in Eqns. (37)-(43) and omitting higher-orders of $\omega$, the following equations are obtained

$$
\begin{aligned}
& \omega f_{j}-\omega f_{j-1}-\frac{h_{j}}{2} \omega u_{j}-\frac{h_{j}}{2} \omega u_{j-1}-\left(e_{1}\right)_{j}=0 \\
& \omega u_{j}-\omega u_{j-1}-\frac{h_{j}}{2} \omega v_{j}-\frac{h_{j}}{2} \omega v_{j-1}-\left(e_{2}\right)_{j}=0 \\
& \omega s_{j}-\omega s_{j-1}-\frac{h_{j}}{2} \omega t_{j}-\frac{h_{j}}{2} \omega t_{j-1}-\left(e_{3}\right)_{j}=0
\end{aligned}
$$




$$
\begin{aligned}
& \omega g_{j}-\omega g_{j-1}-\frac{h_{j}}{2} \omega p_{j}-\frac{h_{j}}{2} \omega p_{j-1}-\left(e_{4}\right)_{j}=0 \\
& \left(a_{1}\right)_{j} \omega v_{j}+\left(a_{2}\right)_{j} \omega v_{j-1}+\left(a_{3}\right)_{j} \omega f_{j}+\left(a_{4}\right)_{j} \omega f_{j-1}+\left(a_{5}\right)_{j} \omega u_{j} \\
& +\left(a_{6}\right)_{j} \omega u_{j-1}+\left(a_{7}\right)_{j} \omega s_{j}+\left(a_{8}\right)_{j} \omega s_{j-1} \\
& +\left(a_{9}\right)_{j} \omega g_{j}+\left(a_{10}\right)_{j} \omega g_{j-1}-\left(e_{5}\right)_{j-1 / 2}=0 \\
& \left(b_{1}\right)_{j} \omega t_{j}+\left(b_{2}\right)_{j} \omega t_{j-1}+\left(b_{3}\right)_{j} \omega f_{j}+\left(b_{4}\right)_{j} \omega f_{j-1} \\
& +\left(b_{5}\right)_{j} \omega u_{j}+\left(b_{6}\right)_{j} \omega u_{j-1}+\left(b_{7}\right)_{j} \omega s_{j}+\left(b_{8}\right)_{j} \omega s_{j-1} \\
& +\left(b_{9}\right)_{j} \omega v_{j}+\left(b_{10}\right)_{j} \omega v_{j-1}+\left(b_{11}\right)_{j} \omega p_{j}+\left(b_{12}\right)_{j} \omega p_{j-1}=0 \\
& \left(c_{1}\right)_{j} \omega p_{j}+\left(c_{2}\right)_{j} \omega p_{j-1}+\left(c_{3}\right)_{j} \omega f_{j}+\left(c_{4}\right)_{j} \omega f_{j-1}+\left(c_{5}\right)_{j} \omega u_{j}+\left(c_{6}\right)_{j} \omega u_{j-1} \\
& +\left(c_{7}\right)_{j} \omega g_{j}+\left(c_{8}\right)_{j} \omega g_{j-1}+\left(c_{9}\right)_{j} \omega t_{j}+\left(c_{10}\right)_{j} \omega t_{j-1}-\left(e_{7}\right)_{j-1 / 2}=0
\end{aligned}
$$

where,

$$
\begin{aligned}
&\left(a_{1}\right)_{j}=\left(1-n_{a}\right) \xi+n_{a} W e \xi v_{j-1 / 2}+h_{j}\left[\frac{1+\alpha}{2} f_{j-1 / 2}-\frac{\alpha}{2} h_{j} f_{j-1 / 2}^{n-1}\right] \\
&\left(a_{2}\right)_{j}=-\left(1-n_{a}\right) \xi-n_{a} W e \xi v_{j-1 / 2}+h_{j}\left[\frac{1+\alpha}{2} f_{j-1 / 2}-\frac{\alpha}{2} h_{j} f_{j-1 / 2}^{n-1}\right] \\
&\left(a_{3}\right)_{j}=h_{j}\left[\frac{(1+\alpha)}{2} v_{j-1 / 2}+\frac{\alpha}{2} v_{j-1 / 2}^{n-1}\right] \\
&\left(a_{4}\right)_{j}=\left(a_{3}\right)_{j}, \\
&\left(a_{5}\right)_{j}=h_{j}\left[-(1+\alpha) u_{j-1 / 2}-\frac{M_{a}}{2} \sin ^{2} \Psi\right] \\
&\left(a_{6}\right)_{j}=\left(a_{5}\right)_{j}, \\
&\left(a_{7}\right)_{j}=h_{j} \frac{\lambda_{c} B}{2}+h_{j} \lambda_{c} B N_{c} s_{j-1 / 2}, \\
&\left(a_{8}\right)_{j}=\left(a_{7}\right)_{j}, \\
&\left(a_{9}\right)_{j}=-\frac{B}{2} h_{j} N_{r}, \\
&\left(a_{10}\right)_{j}=\left(a_{9}\right)_{j}, \\
&\left(b_{1}\right)_{j}=\frac{1}{\operatorname{Pr}}\left[1+\frac{4}{3} R_{a}\right]+h_{j}\left[N_{T} t_{j-1 / 2}+\frac{N_{B}}{2} p_{j-1 / 2}+\frac{1+\alpha}{2} f_{j-1 / 2}-\frac{\alpha}{2} f_{j-1 / 2}^{n-1}\right] \\
&\left(b_{2}\right)_{j}=-\frac{1}{\operatorname{Pr}}\left[1+\frac{4}{3} R_{a}\right]+h_{j}\left[N_{T} t_{j-1 / 2}+\frac{N_{B}}{2} p_{j-1 / 2}+\frac{1+\alpha}{2} f_{j-1 / 2}-\frac{\alpha}{2} f_{j-1 / 2}^{n-1}\right] \\
&\left(b_{3}\right)_{j}=h_{j}\left[\frac{(1+\alpha)}{2} t_{j-1 / 2}+\frac{\alpha}{2} t_{j-1 / 2}^{n-1}\right], \\
&\left(b_{4}\right)_{j}=\left(b_{3}\right)_{j} \\
&\left(b_{5}\right)_{j}=h_{j}\left[M_{a} E_{c} \sin ^{2} \Psi u_{j-1 / 2}-\frac{\alpha}{2} s_{j-1 / 2}+\frac{\alpha}{2} s_{j-1 / 2}^{n-1}\right] \\
&\left(b_{6}\right)_{j}=\left(b_{5}\right)_{j}, \\
&\left(b_{7}\right)_{j}=h_{j}\left[-\frac{\alpha}{2} u_{j-1 / 2}-\frac{\alpha}{2} u_{j-1 / 2}^{n-1}\right] \\
&=\left(b_{7}\right)_{j},
\end{aligned}
$$




$$
\begin{aligned}
& \left(b_{9}\right)_{j}=h_{j}\left[E_{c}\left(1-n_{a}\right) \xi^{2} v_{j-1 / 2}+\frac{E_{c} n_{a} W e}{2} \xi^{3}\left(v_{j-1 / 2}\right)^{2}\right], \\
& \left(b_{10}\right)_{j}=\left(b_{9}\right)_{j} \\
& \left(b_{11}\right)_{j}=h_{j}\left[\frac{N_{B}}{2} t_{j-1 / 2}\right] \text {, } \\
& \left(b_{12}\right)_{j}=h_{j}\left[\frac{N_{B}}{2} t_{j-1 / 2}\right] \\
& \left(c_{1}\right)_{j}=\frac{1}{S c}+h_{j}\left[\frac{(1+\alpha)}{2} f_{j-1 / 2}-\frac{\alpha}{2} f_{j-1 / 2}^{n-1}\right], \\
& \left(c_{2}\right)_{j}=-\frac{1}{S c}+h_{j}\left[\frac{(1+\alpha)}{2} f_{j-1 / 2}-\frac{\alpha}{2} f_{j-1 / 2}^{n-1}\right] \text {, } \\
& \left(c_{3}\right)_{j}=h_{j}\left[\frac{(1+\alpha)}{2} p_{j-1 / 2}+\frac{\alpha}{2} p_{j-1 / 2}^{n-1}\right] \text {, } \\
& \left(c_{4}\right)_{j}=\left(c_{3}\right)_{j} \text {, } \\
& \left(c_{5}\right)_{j}=h_{j}\left[-\frac{\alpha}{2} g_{j-1 / 2}+\frac{\alpha}{2} g_{j-1 / 2}^{n-1}\right] \text {, } \\
& \left(c_{6}\right)_{j}=\left(c_{5}\right)_{j} \text {, } \\
& \left(c_{7}\right)_{j}=h_{j}\left[-\frac{\alpha}{2} u_{j-1 / 2}-\frac{\alpha}{2} u_{j-1 / 2}^{n-1}\right] \text {, } \\
& \left(c_{8}\right)_{j}=\left(c_{7}\right)_{j} \text {, } \\
& \left(c_{9}\right)_{j}=\frac{1}{S c} \frac{N_{T}}{N_{B}}, \\
& \left(c_{10}\right)_{j}=-\left(c_{9}\right)_{j} \text {, } \\
& \left(e_{5}\right)_{j-1 / 2}=\left(v_{j-1}-v_{j}\right)\left[\left(1-n_{a}\right) \xi+n_{a} W e \xi v_{j-1 / 2}\right]-h_{j}(1+\alpha) f_{j-1 / 2} \\
& v_{j-1 / 2}+(1+\alpha) h_{j}\left(u_{j-1 / 2}\right)^{2}+M_{a} h_{j} \sin ^{2} \Psi u_{j-1 / 2}-M_{a} \sin ^{2} \Psi 2 B h_{j} \\
& -2 H h_{j}-\lambda_{c} B h_{j}\left[s_{j-1 / 2}+N_{c}\left(s_{j-1 / 2}\right)^{2}-N_{r} g_{j-1 / 2}\right] \\
& +\alpha h_{j} v_{j-1 / 2} f_{j-1 / 2}^{n-1}-\alpha h_{j} f_{j-1 / 2} v_{j-1 / 2}^{n-1}+\left(E_{1}\right)_{j-1 / 2}^{i-1} \\
& \left(e_{6}\right)_{j-1 / 2}=\left(t_{j-1}-t_{j}\right)\left[\frac{1}{\operatorname{Pr}}\left(1+\frac{4}{3} R_{a}\right)\right]-N_{B} h_{j} t_{j-1 / 2} p_{j-1 / 2}-N_{T} h_{j}\left(t_{j-1 / 2}\right)^{2} \\
& -h_{j} M_{a} \xi^{2} E_{c} \sin ^{2} \Psi\left(u_{j-1 / 2}\right)^{2}-E_{c}\left(1-n_{a}\right) \xi^{2} h_{j}\left(v_{j-1 / 2}\right)^{2}-E_{c} n_{a} \frac{W e}{2} \xi^{3} h_{j}\left(v_{j-1 / 2}\right)^{3} \\
& -(1+\alpha) h_{j} f_{j-1 / 2} t_{j-1 / 2}+h_{j} \alpha u_{j-1 / 2} s_{j-1 / 2}-\alpha h_{j} s_{j-1 / 2}^{n-1} u_{j-1 / 2} \\
& +\alpha h_{j} u_{j-1 / 2}^{n-1} s_{j-1 / 2}+\alpha h_{j} f_{j-1 / 2}^{n-1} t_{j-1 / 2},-\alpha h_{j} t_{j-1 / 2}^{n-1} f_{j-1 / 2} \text {, } \\
& \left(e_{7}\right)_{j-1 / 2}=\frac{1}{S c}\left(p_{j-1}-p_{j}\right)-(1+\alpha) h_{j} f_{j-1 / 2} p_{j-1 / 2} \\
& +h_{j} \alpha u_{j-1 / 2} g_{j-1 / 2}-\alpha h_{j} g_{j-1 / 2}^{n-1} u_{j-1 / 2}+\alpha h_{j} u_{j-1 / 2}^{n-1} g_{j-1 / 2}+\alpha h_{j} f_{j-1 / 2}^{n-1} p_{j-1 / 2}
\end{aligned}
$$




$$
-\alpha h_{j} p_{j-1 / 2}^{n-1} f_{j-1 / 2}+\left[\frac{1}{S c} \frac{N_{T}}{N_{B}}\right]\left(t_{j-1}-t_{j}\right)+\left(E_{3}\right)_{j-1 / 2}^{i-1} .
$$

The boundary conditions Eqn. (36) become

$$
\left.\begin{array}{l}
\omega f_{0}=0, \omega u_{0}=0, \omega s_{0}^{n}=0, \omega g_{0}=0 \\
\omega u_{J}=0, \omega s_{J}=0, \omega g_{J}=0, f_{0}^{n}=u_{0}^{n}=0 \\
s_{0}^{n}=1, \quad g_{0}^{n}=1, \quad u_{J}^{n}=0, \quad s_{J}^{n}=0, g_{J}^{n}=0
\end{array}\right\}
$$

\section{Step 4:}

The block-elimination method is used to compute the linearized difference Eqns. (47)-(56) which is outlined by Cebeci and Pradshaw [42].

$$
A \omega=e
$$

where,

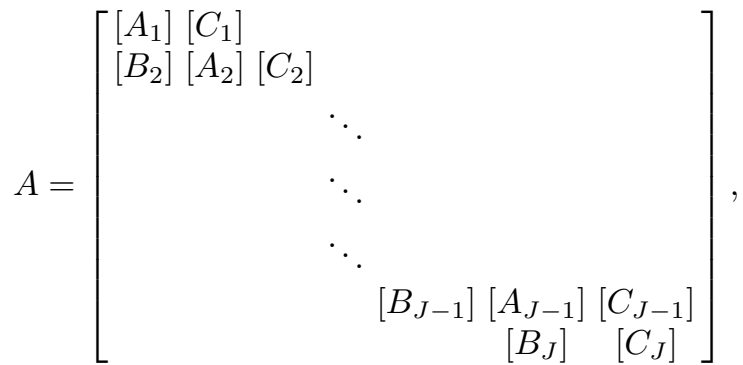

$$
\begin{aligned}
& \omega=\left[\begin{array}{c}
{\left[\omega_{1}\right]} \\
{\left[\omega_{2}\right]} \\
\vdots \\
{\left[\omega_{J-1}\right]} \\
{\left[\omega_{J}\right]}
\end{array}\right], \quad e=\left[\begin{array}{c}
{\left[e_{1}\right]} \\
{\left[e_{2}\right]} \\
\\
\vdots \\
\\
{\left[e_{J-1}\right]} \\
{\left[e_{J}\right]}
\end{array}\right] .
\end{aligned}
$$

The elements of the matrices are as follows

$$
\left[A_{1}\right]=\left[\begin{array}{ccccccc}
0 & 0 & 0 & 1 & 0 & 0 & 0 \\
-\frac{h_{j}}{2} & 0 & 0 & 0 & -\frac{h_{j}}{2} & 0 & 0 \\
0 & -\frac{h_{j}}{2} & 0 & 0 & 0 & -\frac{h_{j}}{2} & 0 \\
0 & 0 & -\frac{h_{j}}{2} & 0 & 0 & 0 & -\frac{h_{j}}{2} \\
\left(a_{2}\right)_{1} & 0 & 0 & \left(a_{3}\right)_{1} & \left(a_{1}\right)_{1} & 0 & 0 \\
\left(b_{10}\right)_{1} & \left(b_{12}\right)_{1} & \left(b_{2}\right)_{1} & \left(b_{3}\right)_{1} & \left(b_{9}\right)_{1} & \left(b_{11}\right)_{1} & \left(b_{1}\right)_{1} \\
0 & \left(c_{2}\right)_{1} & \left(c_{10}\right)_{1} & \left(c_{3}\right)_{1} & 0 & \left(c_{1}\right)_{1} & \left(c_{9}\right)_{1}
\end{array}\right],
$$




$$
\begin{aligned}
& {\left[A_{J}\right]=\left[\begin{array}{ccccccc}
-\frac{h_{j}}{2} & 0 & 0 & 1 & 0 & 0 & 0 \\
-1 & 0 & 0 & 0 & -\frac{h_{j}}{2} & 0 & 0 \\
0 & -1 & 0 & 0 & 0 & -\frac{h_{j}}{2} & 0 \\
0 & 0 & -1 & 0 & 0 & 0 & -\frac{h_{j}}{2} \\
\left(a_{6}\right)_{j}\left(a_{10}\right)_{j} & \left(a_{8}\right)_{j} & \left(a_{3}\right)_{j}\left(a_{1}\right)_{j} & 0 & 0 \\
\left(b_{6}\right)_{j} & 0 & \left(b_{8}\right)_{j} & \left(b_{3}\right)_{j} & \left(b_{9}\right)_{j} & \left(b_{11}\right)_{j} & \left(b_{1}\right)_{j} \\
\left(c_{6}\right)_{j} & \left(c_{8}\right)_{j} & 0 & \left(c_{3}\right)_{j} & 0 & \left(c_{1}\right)_{j} & \left(c_{9}\right)_{j}
\end{array}\right], 2 \leq j \leq J} \\
& {\left[B_{J}\right]=\left[\begin{array}{ccccccc}
0 & 0 & 0 & -1 & 0 & 0 & 0 \\
0 & 0 & 0 & 0 & -\frac{h_{j}}{2} & 0 & 0 \\
0 & 0 & 0 & 0 & 0 & -\frac{h_{j}}{2} & 0 \\
0 & 0 & 0 & 0 & 0 & 0 & -\frac{h_{j}}{2} \\
0 & 0 & 0 & \left(a_{4}\right)_{j} & \left(a_{2}\right)_{j} & 0 & 0 \\
0 & 0 & 0 & \left(b_{4}\right)_{j}\left(b_{10}\right)_{j} & \left(b_{12}\right)_{j} & \left(b_{2}\right)_{j} \\
0 & 0 & 0 & \left(c_{4}\right)_{j} & 0 & \left(c_{2}\right)_{j} & \left(c_{10}\right)_{j}
\end{array}\right], 2 \leq j \leq J} \\
& {\left[C_{J}\right]=\left[\begin{array}{ccccccc}
-\frac{h_{j}}{2} & 0 & 0 & 0 & 0 & 0 & 0 \\
1 & 0 & 0 & 0 & 0 & 0 & 0 \\
0 & 1 & 0 & 0 & 0 & 0 & 0 \\
0 & 0 & 1 & 0 & 0 & 0 & 0 \\
\left(a_{5}\right)_{j}\left(a_{9}\right)_{j}\left(a_{7}\right)_{j} & 0 & 0 & 0 & 0 \\
\left(b_{5}\right)_{j} & 0 & \left(b_{7}\right)_{j} & 0 & 0 & 0 & 0 \\
\left(c_{5}\right)_{j}\left(c_{7}\right)_{j} & 0 & 0 & 0 & 0 & 0
\end{array}\right], 1 \leq j \leq J-1} \\
& {\left[\omega_{1}\right]=\left[\begin{array}{c}
\omega v_{0} \\
\omega p_{0} \\
\omega t_{0} \\
\omega f_{1} \\
\omega v_{1} \\
\omega p_{1} \\
\omega t_{1}
\end{array}\right]} \\
& {\left[\omega_{j}\right]=\left[\begin{array}{c}
\omega u_{j-1} \\
\omega g_{j-1} \\
\omega s_{j-1} \\
\omega f_{j} \\
\omega v_{j} \\
\omega p_{j} \\
\delta t_{j}
\end{array}\right], 2 \leq j \leq J} \\
& \text { and }\left[e_{j}\right]=\left[\begin{array}{c}
\left(e_{1}\right)_{j-(1 / 2)} \\
\left(e_{2}\right)_{j-(1 / 2)} \\
\left(e_{3}\right)_{j-(1 / 2)} \\
\left(e_{4}\right)_{j-(1 / 2)} \\
\left(e_{5}\right)_{j-(1 / 2)} \\
\left(e_{6}\right)_{j-(1 / 2)} \\
\left(e_{7}\right)_{j-(1 / 2)}
\end{array}\right], 1 \leq j \leq J
\end{aligned}
$$

In the present problem, we have fastened the $\xi$ and $\eta$ maximums as 1 and 30 , respectively. The difference between the special nodes is taken as 0.05 


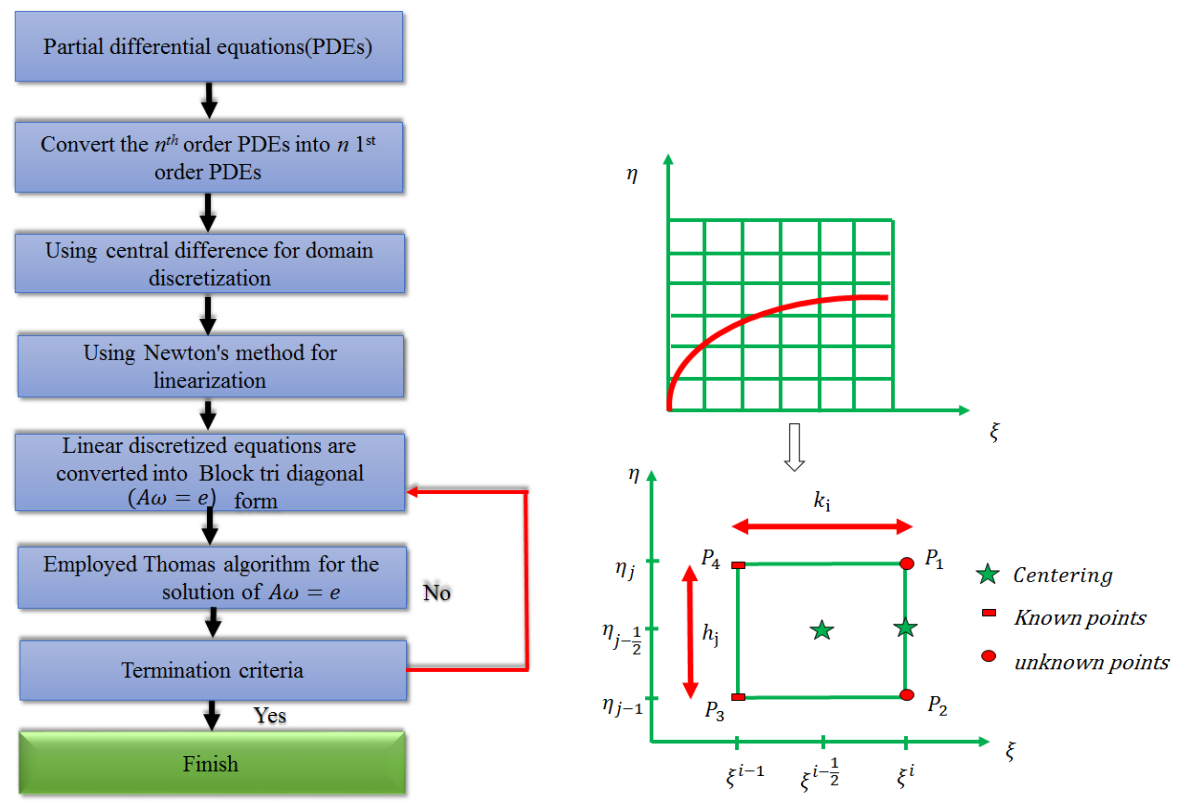

Fig. 2 Solution approach by computer, boundary layer mesh and Keller box element .

in both directions for the solution convergence. It is noteworthy to mention that the parabolic boundary layer is meshed as $(20 \times 600)$. To increase the accuracy of the present solution, the convergence criterion is fixed as $10^{-6}$ at all gird points. Comparison results of $N u^{*}$ for various values of $\xi$ are illustrated in Table 1. It is observed from this table that present results show a good accordance with the results obtained by Merkin [40], Yih[41], and Prasad et al.[22] which depicts that the obtained numerical solution is precise. Tables 2, 3 and 4 manifest the skin friction factor, rate of heat and mass transfer of active parameters. Figure 2 displays the solution approach by computer, boundary layer mesh and Keller box element.

\section{Results and discussion}

This current section is aimed to visualize the influence of emerging parameters like Weissenberg number $(W e=0,1,2,3)$, power law index $\left(n_{a}=\right.$ $0.1,0.3,0.5,0.7)$, magnetic field $\left(M_{a}=0.5,1.0,1.5,2.0\right)$, angle of inclination $\left(\Psi=\frac{\pi}{5}, \frac{\pi}{4}, \frac{\pi}{3}, \frac{\pi}{2}\right)$, mixed convection parameter $\left(\lambda_{c}=1,3,7,10\right)$, Eckert number $\left(E_{c}=0.01,0.1,0.3,0.5\right)$, thermal convention parameter $\left(N_{c}=0.1,0.5,1,1.5\right)$, Brownian motion $\left(N_{B}=0.3,0.5,0.7,0.9\right)$, thermophoresis $\left(N_{T}=0.01,0.1,0.3,0.5\right)$ and radiation $\left(R_{a}=0.0,0.5,1.0,1.5\right)$ on velocity $\left(f^{\prime}\right)$, temperature $(\theta)$, concentration $(\phi)$, skin friction factor $\left(C_{f}^{*}\right)$, rate of heat transfer $\left(N u^{*}\right)$ and rate of mass transfer $\left(S h^{*}\right)$. Dimensionless form of the flow and transport equa- 


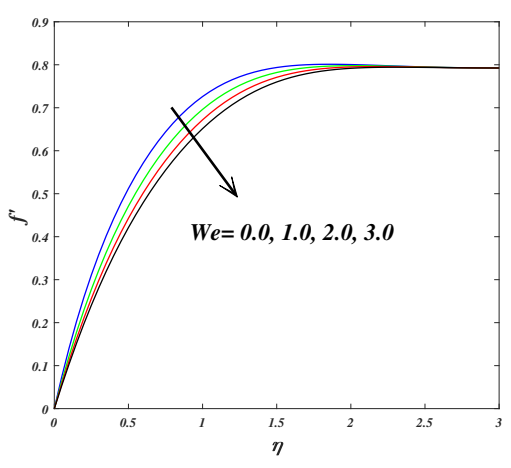

Fig. $3 f^{\prime}$ for uplifting values of $W e$.

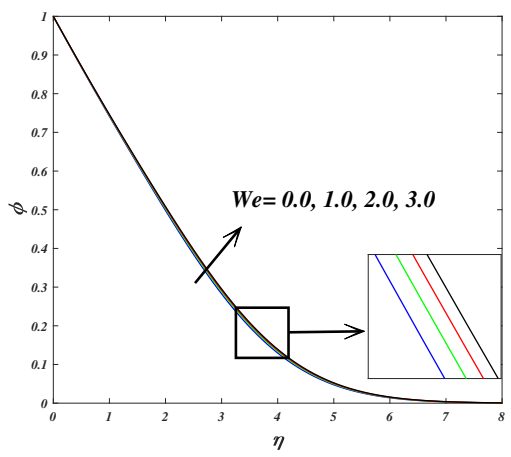

Fig. $5 \phi$ for uplifting values of $W e$.

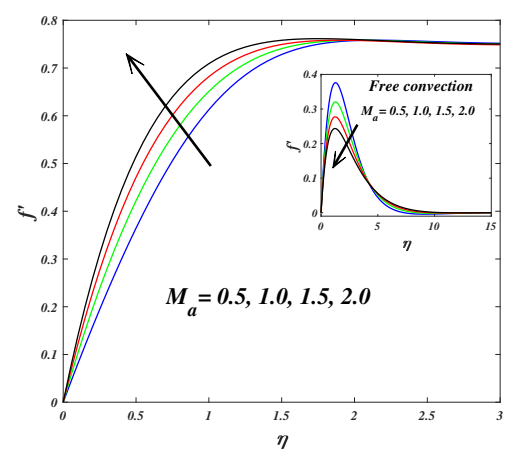

Fig. $7 f^{\prime}$ for uplifting values of $M_{a}$.

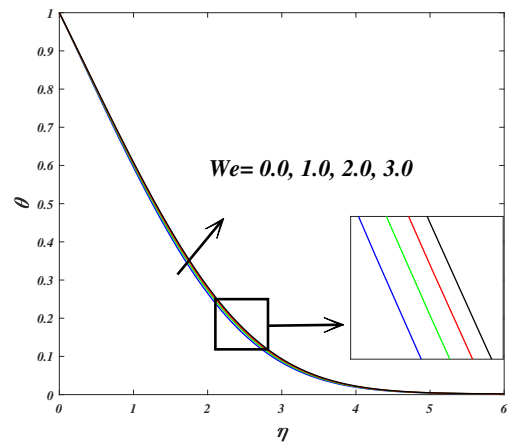

Fig. $4 \theta$ for uplifting values of $W e$.

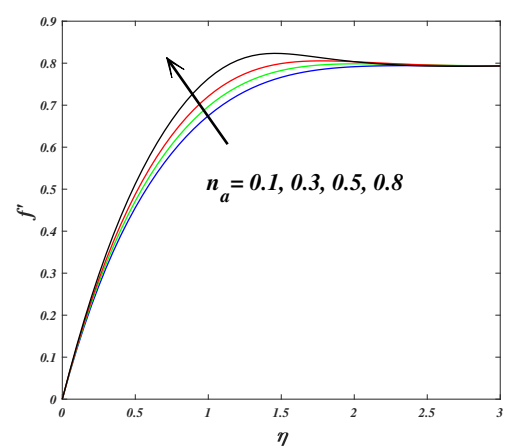

Fig. $6 f^{\prime}$ for uplifting values of $n_{a}$.

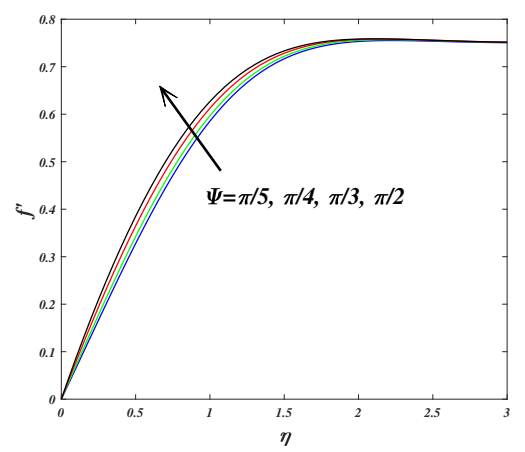

Fig. $8 f^{\prime}$ for uplifting values of $\Psi$.

tions subject to boundary conditions have been computed by KBM. In this computation, the pertinent parameters of tangent hyperbolic nanofluid transport equations are fastened as $N_{B}=0.3, N_{T}=0.1, R_{a}=0.5, E_{c}=0.01, N_{r}=0.5$, $\operatorname{Pr}=1, S c=0.3, M_{a}=0.5, N_{a}=0.3, W e=1.0, \Psi=\frac{\pi}{3}, \lambda_{c}=1$ and $N_{c}=0.1$. In en- 


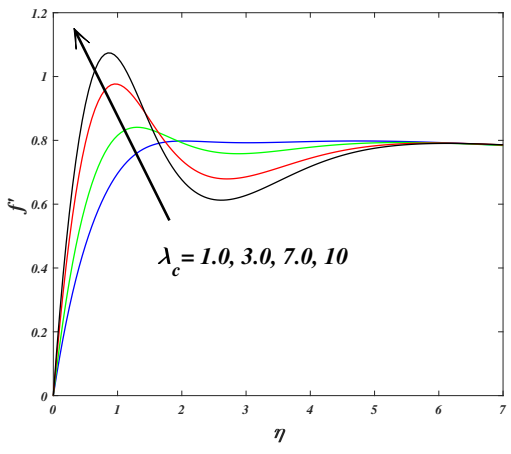

Fig. $9 f^{\prime}$ for uplifting values of $\lambda_{c}$.

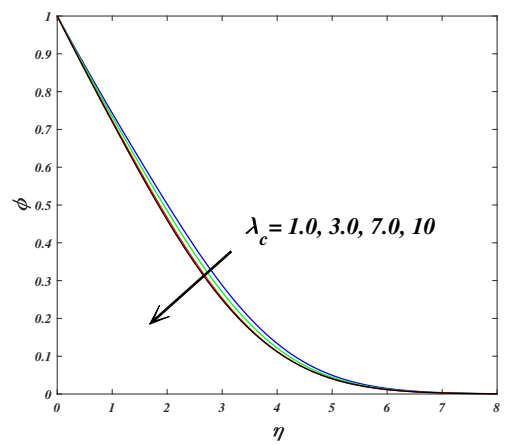

Fig. $11 \phi$ for uplifting values of $\lambda_{c}$.

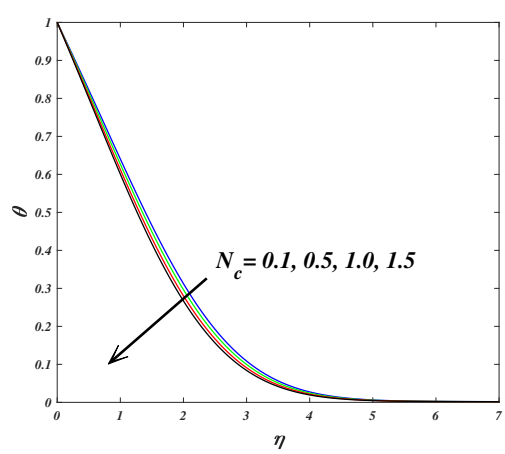

Fig. $13 \theta$ for uplifting values of $N_{c}$.

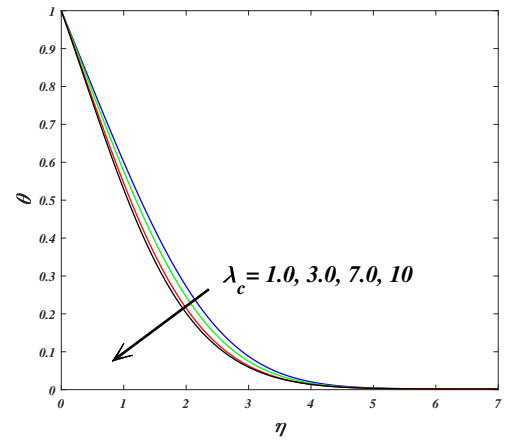

Fig. $10 \theta$ for uplifting values of $\lambda_{c}$.

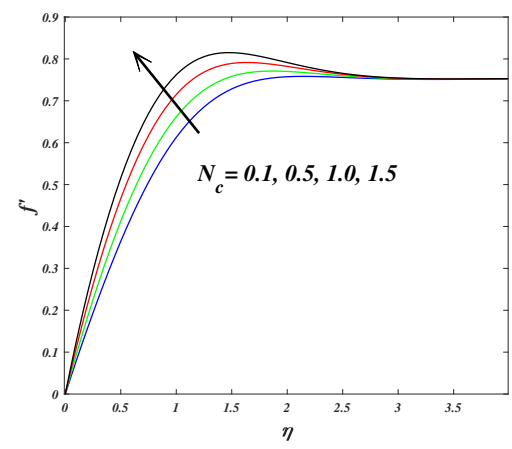

Fig. $12 f^{\prime}$ for uplifting values of $N_{c}$.

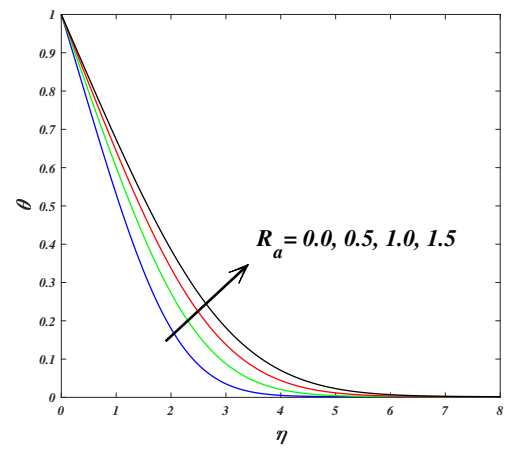

Fig. $14 \theta$ for uplifting values of $R_{a}$.

gineering systems, entropy generation has notable applications to model the energy-efficient devices. The entropy generation minimization approach is very important to illustrate the losses in thermo hydraulic systems and engineering systems. Therefore, this analysis can provide a clear view of researchers to en- 


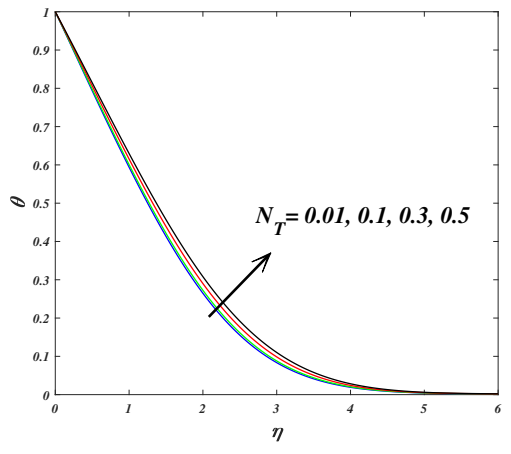

Fig. $15 \theta$ for uplifting values of $N_{T}$.

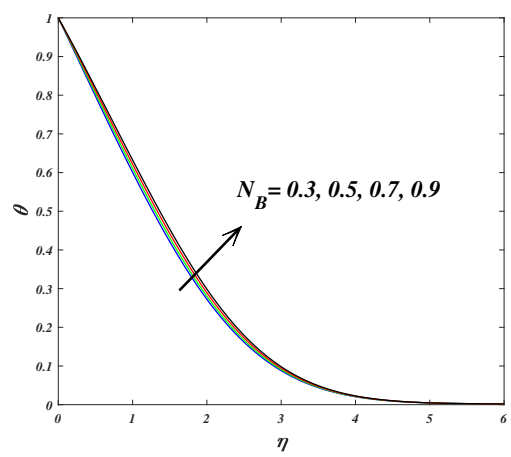

Fig. $17 \theta$ for uplifting values of $N_{B}$.

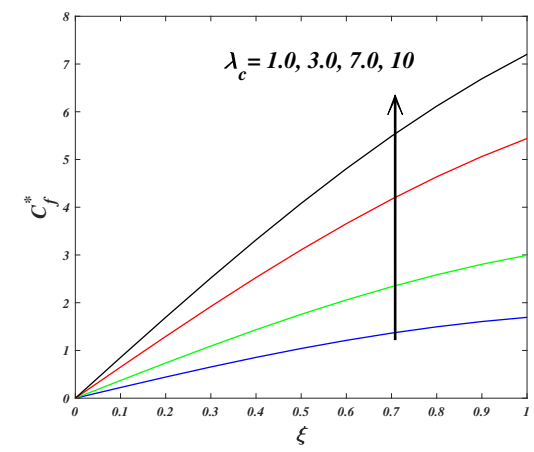

Fig. $19 C_{f}{ }^{*}$ for uplifting values of $\lambda_{c}$.

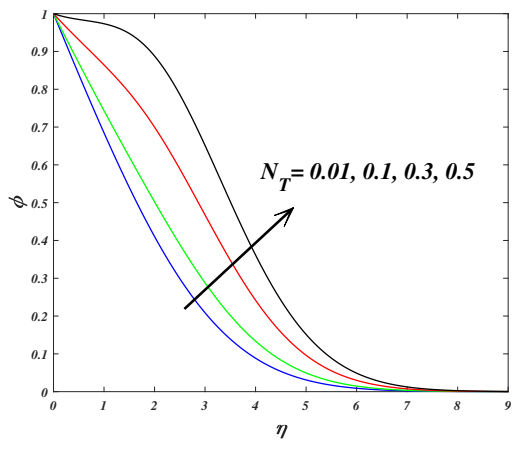

Fig. $16 \phi$ for uplifting values of $N_{T}$.

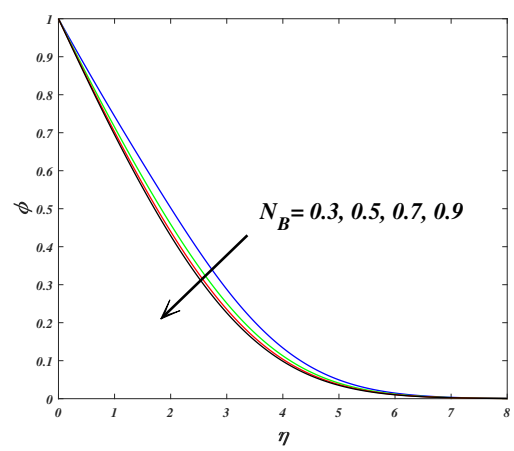

Fig. $18 \phi$ for uplifting values of $N_{B}$.

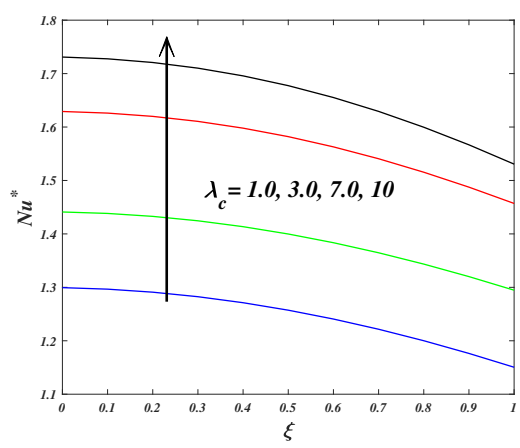

Fig. $20 N u^{*}$ for uplifting values of $\lambda_{c}$.

hance the efficiency of thermal systems. Figures 3-24 depict the characteristics of fluid transport properties, Figs. 25-27 illustrate the streamlines, isotherms and isoconcentrations, and Figs. 28-35 express the entropy generation. 


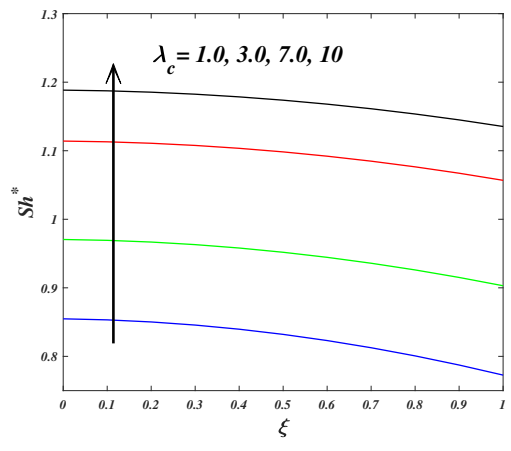

Fig. $21 S h^{*}$ for uplifting values of $\lambda_{c}$.

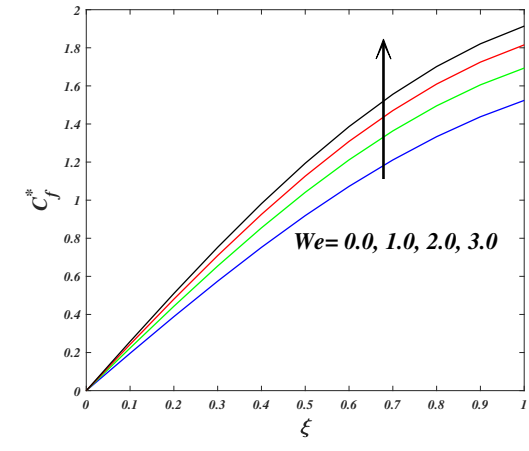

Fig. $22 C_{f}^{*}$ for uplifting values of $W e$.

Figure 3 illustrates the impact of $W e$ on tangent hyperbolic nanofluid velocity. $W e$ is expressed as the ratio of tangent hyperbolic fluid relaxation time and specific process. It is noticed that the relaxation time of the fluid uplifts by enhancing the $W e$. This generates resistance in the fluid flow direction which diminishes the velocity. Figures 4 and 5 exhibit the behavior of $W e$ on temperature and concentration, respectively. From these figures, it is seen that the thermal and mass related boundary layer thicknesses are slightly amplified as $W e$ increases. Hence, tangent hyperbolic nanofluid temperature and concentration distributions raise. Figure 6 depicts the impact of $n_{a}$ on $f^{\prime}$. Increasing values of $n_{a}$ abridge the friction in tangent hyperbolic nanofluid flow which results in uplifting velocity distribution. Impact of $M_{a}$ on tangent hyperbolic nanofluid velocity is depicted in Fig. 7 for nonlinear mixed convection and free convection cases. In an electrically conductive fluid, the magnetic field acting in the transverse to the geometry creates a resistive force called Lorentz force. It is noteworthy to mention that the Lorentz force has the behavior to prohibit fluid velocity, this nature can be observed in free convection flows. To express the nature of free convection in the present problem, the external force and nonlinear convection in the momentum equation (Eqn. 13) are modified as $\left(1-n_{a}\right) f^{\prime \prime \prime}+n_{a} W e f^{\prime \prime \prime} f^{\prime \prime}+f f^{\prime \prime}-\left(f^{\prime}\right)^{2}-M_{a} \sin ^{2} \Psi+\frac{\sin \xi}{\xi}\left(\theta-N_{r} \phi\right)=$ $\xi\left[f^{\prime} \frac{\partial f^{\prime}}{\partial \xi}-f^{\prime \prime} \frac{\partial f}{\partial \xi}\right]$ and the momentum boundary condition (Eqn. 16) is modified as $f^{\prime}=0$ as $\eta \rightarrow \infty$. The usual nature of the magnetic field is obtained in free convection flow which is displayed in the subgraph but the velocity profiles uplift with an increase in $M_{a}$ due to the presence of nonlinear mixed convection. Figure 8 presents the effect of variation of $\Psi$ on nanofluid velocity profiles. It is observed form this figure that direction of the applied magnetic field to the flow shifts from transverse to horizontal for raising the angle of inclination. It is well known that the magnetic field applied in horizontal direction experiences lower Lorentz force compared to vertical direction. In the nonlinear mixed convection flow, fluid velocity elevates for amplifying the an- 
gle of inclination.

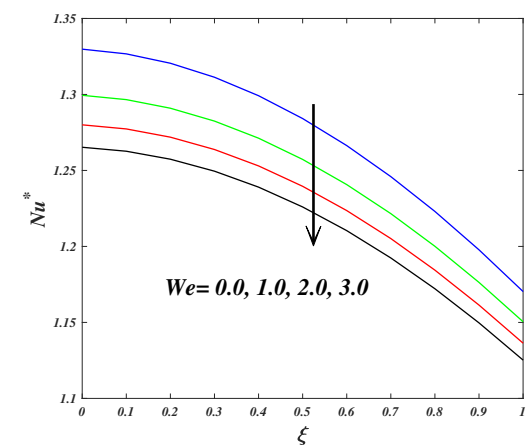

Fig. $23 N u^{*}$ for uplifting values of $W e$

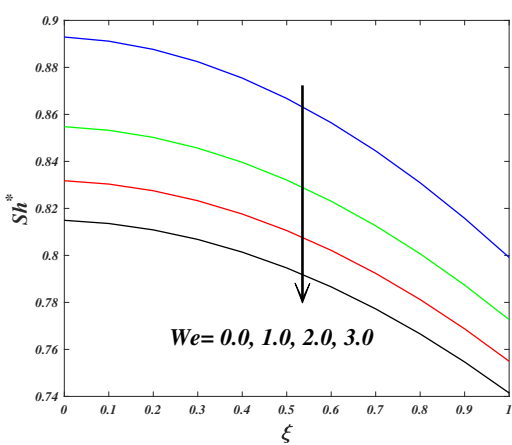

Fig. $24 S h^{*}$ for uplifting values of $W e$.

$f^{\prime}, \theta$ and $\phi$ for several values of $\lambda_{c}$ are manifested in Figs. 9-11. It is noteworthy to mention that the positive and negative values of $\lambda_{c}$ represent the hot and cold cylinder, respectively. It is seen from Fig. 9 that nanofluid velocity shows an increasing nature near the geometry $(\eta<2)$ for increasing the values of $\lambda_{c}$ but the opposite nature is observed in the region $2<\eta<5$. It is clear from Figs. 10 and 11 that nanofluid temperature and concentration
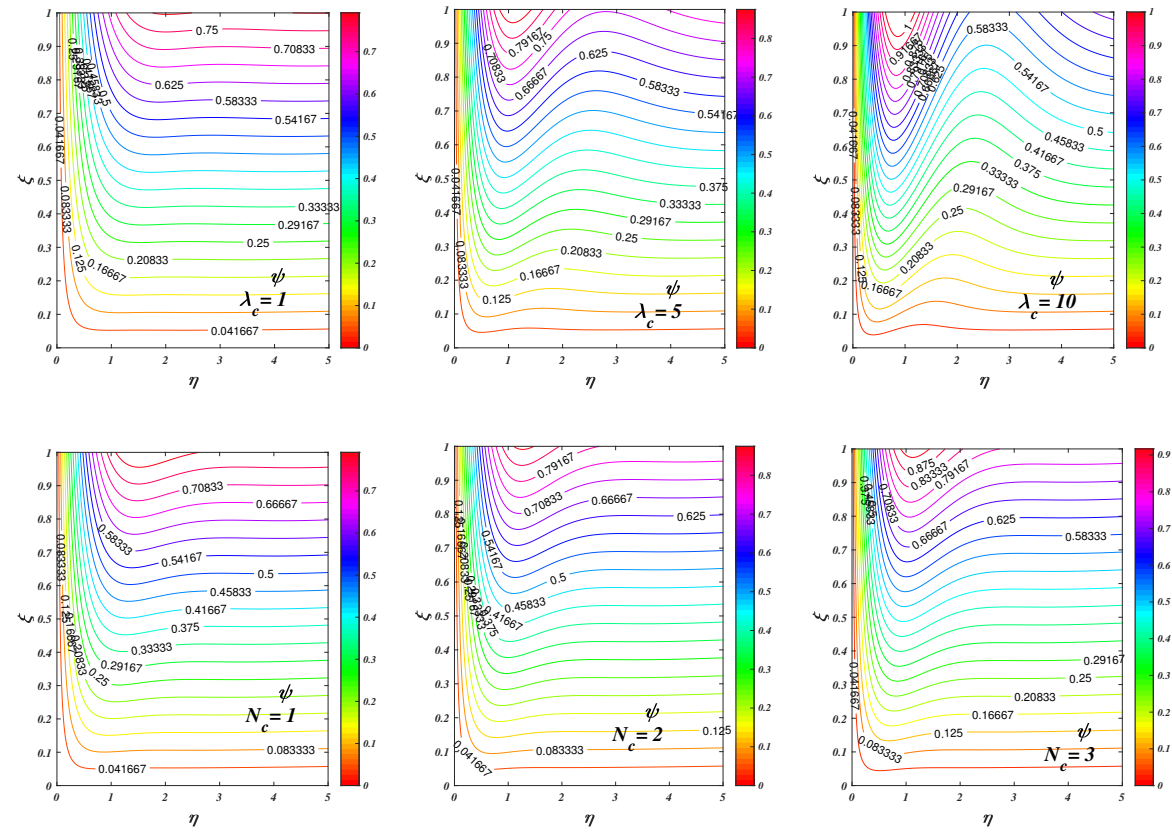

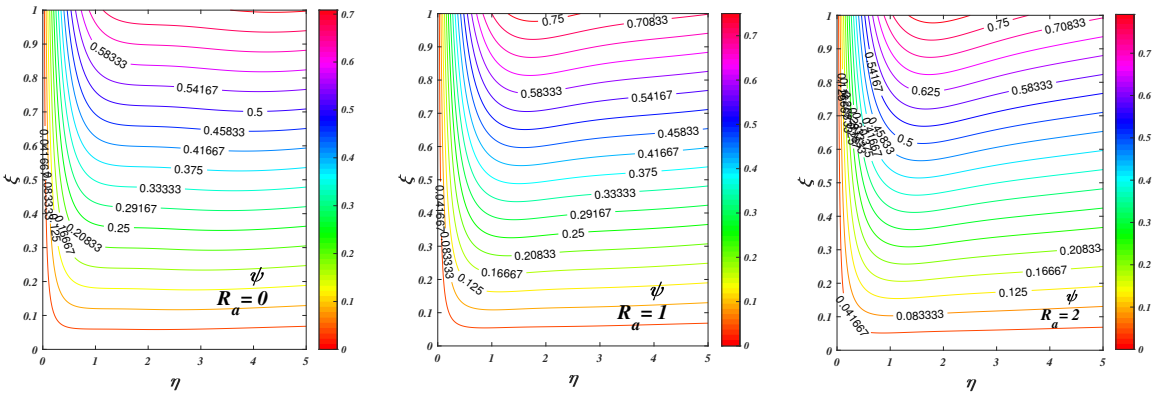

Fig. 25 Streamlines for uplifting values of $\lambda_{c}, N_{c}$ and $R_{a}$.
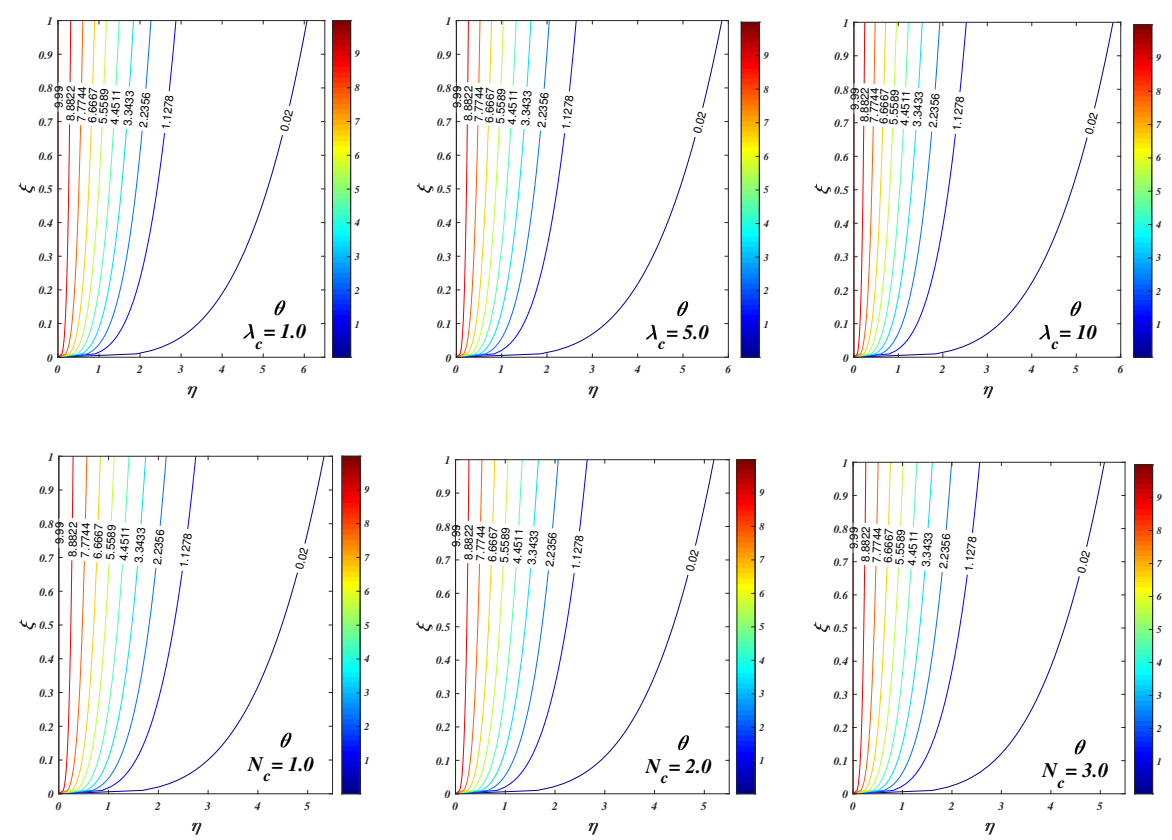

distributions diminish for amplifying the values of $\lambda_{c}$. Physically, higher values of convection parameter tend to enhance the convection cooling effect, as a consequence, nanofluid velocity uplifts. Further, magnification in convection cooling effect causes the decay in temperature and concentration distributions. Figures 12 and 13 are plotted to observe the $f^{\prime}$ and $\theta$ of nanofluid with increasing values of $N_{c}$. It is observed that the intensifying values of $N_{c}$ uplift the nanofluid velocity but diminish the thermal related boundary thickness. This mechanism is related to the buoyancy force, thus increasing values of $N_{c}$ use to strengthen the buoyancy force. This magnification in buoyancy force declines nanofluid temperature distribution. Figure 14 divulges the influence of $R_{a}$ on tangent hyperbolic nanofluid temperature profiles. It is noticed that larger values of radiation lead to elevate the nanofluid temperature. The magnification 

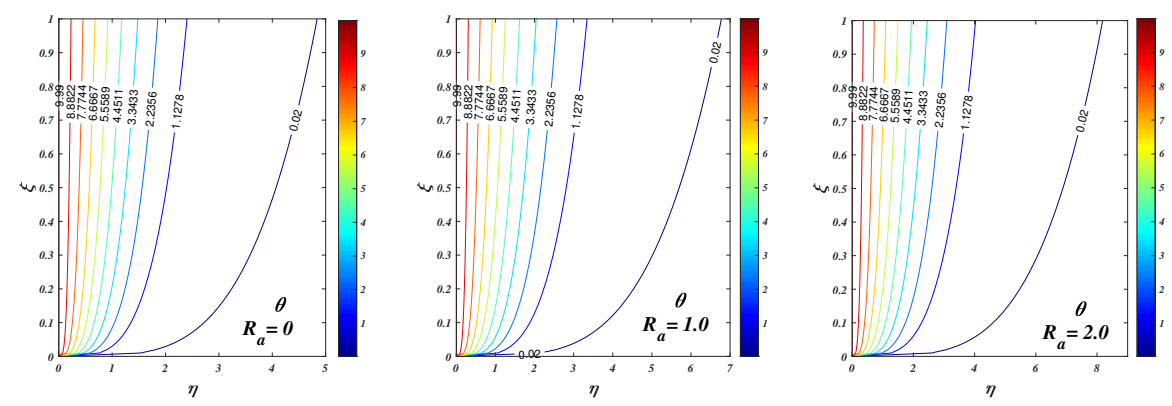

Fig. 26 Isotherms for uplifting values of $\lambda_{c}, N_{c}$ and $R_{a}$.

in radiation motivates the heat transfer proceess in the tangent hyperbolic nanofluid which increase the thermal boundary layer thickness.

Figure 15 displays the results on $\theta$ for distinct values of $N_{T}$. Physically, the phenomenon of thermophoresis is related to the motion of nano-sized solid particles owing to difference in temperature distribution. Larger values of thermophoresis greatly promote the movement of nano-sized solid particles, thus nanofluid temperature rises. Figure 16 exhibits the changes in $\phi$ for several values of $N_{T}$. It is well known that thermophoresis has the nature to displace the nano-sized solid particles from hot place to cool place. This results in an uneven distribution in nano-size particles concentration. So that concentration profile is rising by increasing thermophoresis. The role of $N_{B}$ on tangent hyperbolic nanofluid temperature is displayed in Fig. 17. As the Brownian motion enhances, the nanoparticles move freely and improperly in the fluid which in turn causes the temperature enhancement. The effect of $N_{B}$ on tangent hyperbolic nanofluid concentration is exhibited in Fig. 18. Brownian motion is expressed as the ratio between mass species and nano-sized solid particles. Larger values of Brownian motion diminish the nano-sized solid particles concentration so that the nanofluid concentration decreases.

Figure 19 is drawn to explore the influence of $\lambda_{c}$ on the skin friction factor. It is noticed that the skin friction factor is 0 at $\xi=0$ and the skin friction factor is raised when $\xi$ enhances. Moreover, an increase in mixed convection parameter augments the skin friction factor of nanofluid at the surface. Figures 20 and 21 are plotted to examine the impact of mixed convection parameter on rate of heat and mass transfer. It is observed that the rate of heat and mass transfer of nanofluid at the surface upsurge by magnifying the mixed convection. The impact of $W e$ on the skin friction factor, rate of heat and mass transfer are elucidated in Figs 22-24, respectively. It is seen that the skin friction factor at the surface enhances with an increment in $W e$ but the reverse behavior is exhibited on the rate of heat and mass transfer. It is to be noted that magnitude of rate of heat and mass transfer near the surface is higher while the rate of heat and mass transfer decline for increasing the height of 

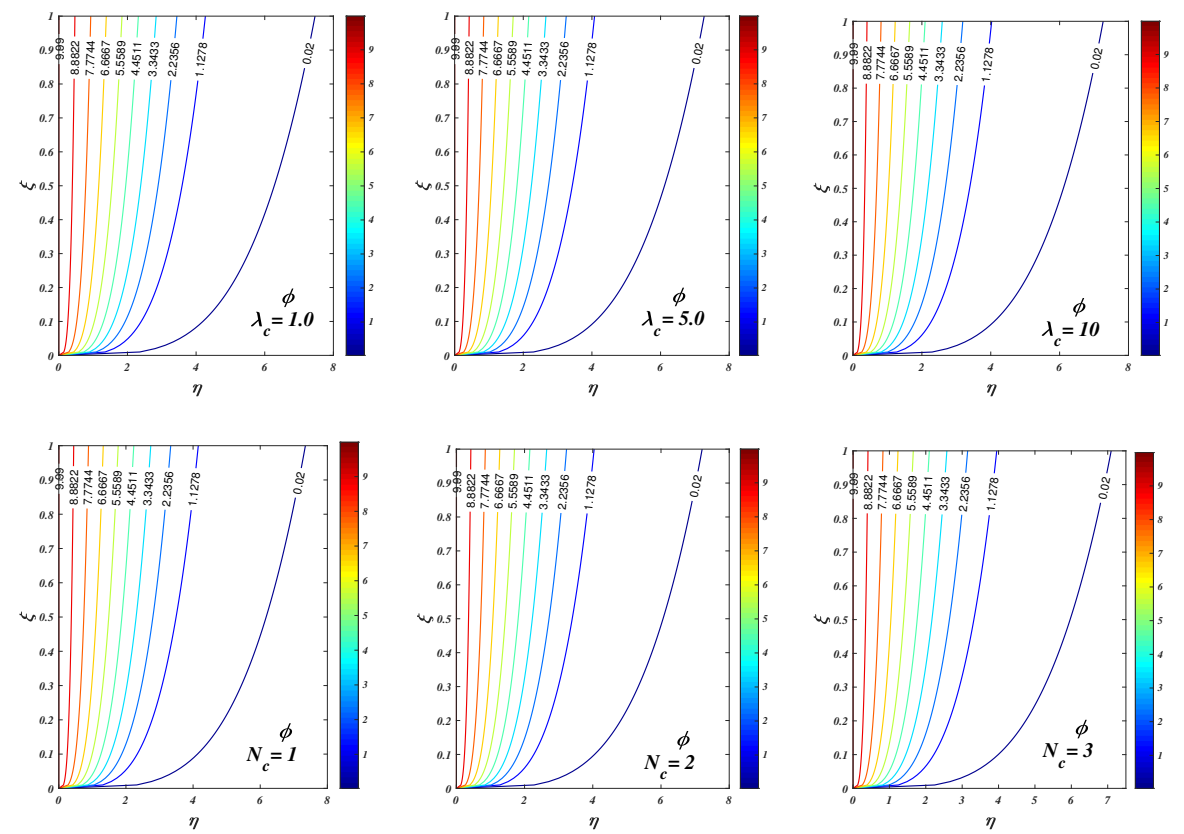

the wall.

Figures 25-27 are plotted in order to illustrate the streamlines, isotherms and isoconcentrations for various values of $\lambda_{c}, N_{c}$ and $R_{a}$. The influence of $\lambda_{c}$, $N_{c}$ and $R_{a}$ on streamline is demonstrated in Fig. 25. It is observed from these figures that the streamlines are more dense near the wall at $\xi<1$. However, streamlines manifest an oscillating nature for increasing the $\lambda_{c}$ from 1 to 10 . Moreover, for higher values of $N_{c}$ and $R_{a}$, the streamlines show the oscillating nature due to the buoyancy force. Figures 26 and 27 are manifested the isotherms and isoconcentrations for distinct values of $\lambda_{c}, N_{c}$ and $R_{a}$, respectively. It is noticed from these figures that isotherms and isoconcentrations thicknesses slightly decrease with an increment in $\lambda_{c}$ and $N_{c}$. However, higher values of $R_{a}$ enhance the isotherms and isoconcentrations.

\subsection{Entropy generation}

This numerical study is the analysis of entropy generation in a tangent hyperbolic non-Newtonian nanofluid over a normal surface of a circular cylinder. The future examines will exhibits entropy generation in a various nonNewtonian nanofluid (eg. Williamson, Maxwell, Jeffrey ). Furthermore, the rough surface of a circular cylinder also will be taken for the analysis. Characteristics of entropy generation $\left(N_{G}\right)$ and Bejan number $(B e)$ under the impacts radiation $\left(R_{a}=0.0,0.5,1.0,1.5\right)$, Brinkman number $(B r=0.1,0.3,0.5,0.5)$, 

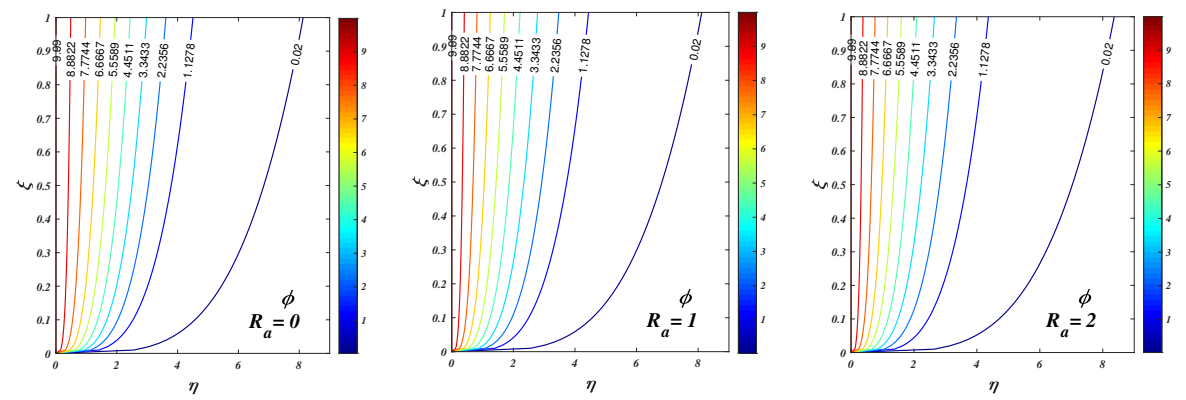

Fig. 27 Isoconcentrations for uplifting values of $\lambda_{c}, N_{c}$ and $R_{a}$.

mixed convection $\left(\lambda_{c}=1,3,7,10\right)$, dimensionless temperature ratio variable $\left(\alpha_{T}=0.1,0.5,1.0,1.5\right)$, Eckert number $\left(E_{c}=0.01,0.1,0.3,0.5\right)$ and thermal convection $\left(N_{c}=0.1,0.5,1.0,1.5\right)$ are explored graphically in Figs. 28-35. Figure 28 elucidates the influence of $R_{a}$ on $N_{G}$. There is an augment in $N_{G}$ for raising values of $R_{a}$. As a result of $R_{a}$, the internal energy of the hyperbolic nanofluid flow system is uplifted which corresponds to enhance $N_{G}$. Figure 29 represents the effect of $\alpha_{T}$ on $N_{G}$. It is observed from this figure that there is a raise in $N_{G}$ with an increment in $\alpha_{T} . \alpha_{T}$ significantly controls the fluid frictional effects, thus enhances the $N_{G}$. The behavior of $E_{c}$ on $N_{G}$ is manifested in Fig. 30. Physically, $E_{c}$ is related to self-heating mechanism of the nanofluids due to the influence of dissipation. It is seen that $N_{G}$ decreases for uplifting values of $E_{c}$. Figure 31 is plotted to scrutinize the changes in $N_{G}$ with increasing values of $\lambda_{c}$. It is evident that an increment in $\lambda_{c}$ promotes the rate of heat transfer and generate more heat, as a consequence $N_{G}$ enhances near the geometry $(\eta<2)$ but an opposite trend is manifested in the region $(4<\eta<4.5)$. Figures 32 and 33 show the effect of $N_{c}$ on $N_{G}$ and $B e$, respectively. It can be seen that the effect of $N_{c}$ enhances the total entropy generation while the reverse trend is observed on $\mathrm{Be}$. As a consequence of buoyancy force, $N_{G}$ and $B e$ express this nature. Figure 34 displays $N_{G}$ for varying $\Psi$. It is evident that increasing values of $\Psi$ tend to lessen $B e$. The variation in $B e$ with raising values of $B r$ is manifested in Fig. 35. This figure reveals that $B e$ decreases for uplifting values of $B r$. It is known that $B r$ is the relationship between heat and molecular transport caused by fluid friction and heat transfer. Generally, $\mathrm{Br}$ admits viscous heating corresponding with the convective heat transfer. However, when viscous effects produce more heat which intend to control heat transport via molecular conduction. This is the reason for reduction in $\mathrm{Be}$. 


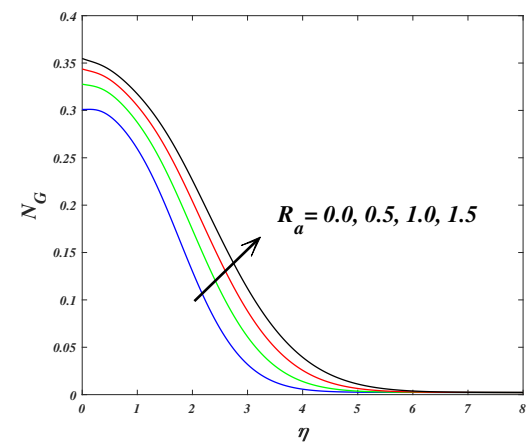

Fig. $28 N_{G}$ for uplifting values of $R_{a}$.

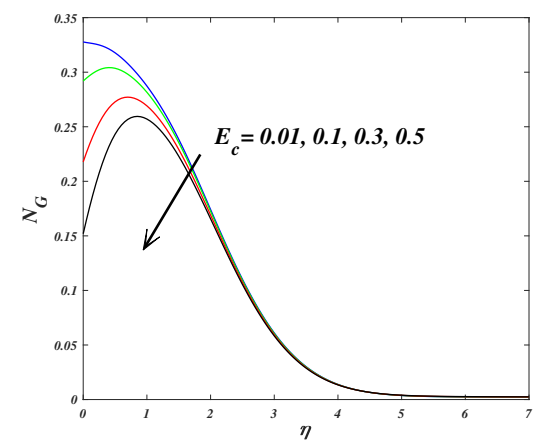

Fig. $30 N_{G}$ for uplifting values of $E_{c}$.

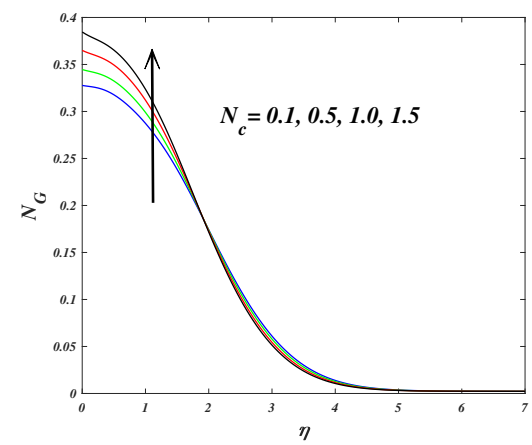

Fig. $32 N_{G}$ for uplifting values of $N_{c}$.

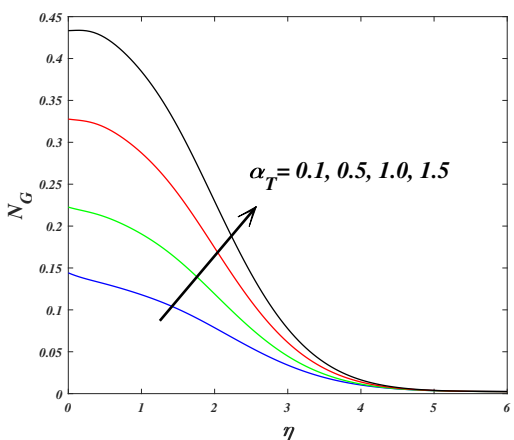

Fig. $29 N_{G}$ for uplifting values of $\alpha_{T}$.

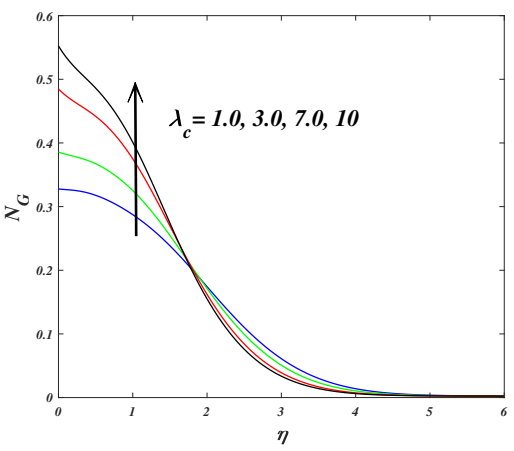

Fig. $31 N_{G}$ for uplifting values of $\lambda_{c}$.

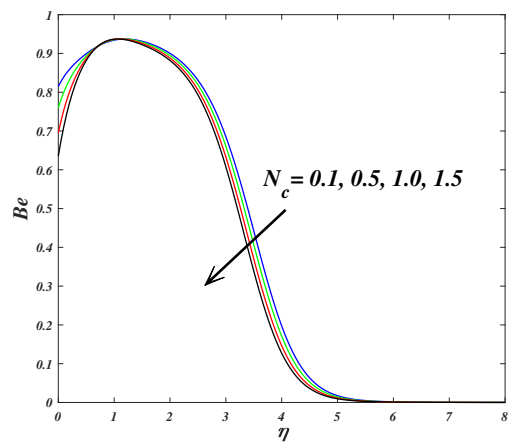

Fig. $33 B e$ for uplifting values of $N_{c}$.

\section{Conclusion}

This study has been presented to explore the heat and mass transfer characteristics and entropy analysis on hyperbolic tangent nanofluid over a circular 


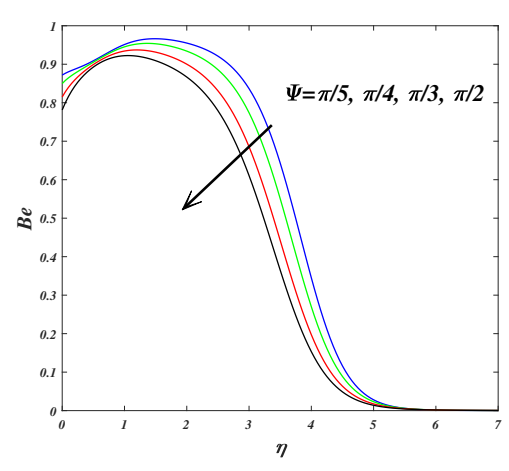

Fig. $34 B e$ for uplifting values of $\Psi$.

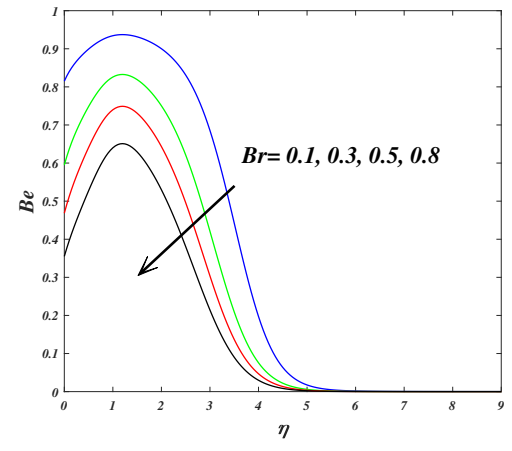

Fig. $35 B e$ for uplifting values of $B r$.

cylinder with the impacts of nonlinear Boussinesq approximation and inclined magnetic field. Non-similar variables are utilized to transform the dimensional equations into a dimensionless form which are computed by adopting an implicit finite difference KBM method. The outcomes are demonstrated in terms of a two-dimensional plot, streamlines, isotherms and isoconcentrations contours. The main findings of the current study are listed below.

- Tangent hyperbolic nanofluid velocity uplifts for enhancing the mixed convection parameter.

- The velocity and temperature distributions show an opposite nature due to the increase in thermal convection parameter.

- Temperature and concentration distributions raise with an increment in the Weissenberg number.

- The rate of heat and mass transfer at $\xi=0$ is higher than at $\xi=1$.

- Higher values of radiation, mixed convection and thermal convection parameters augment the total entropy generation.

- Magnifications in angle of inclination and Brinkman number reduce the Bejan number.

- Streamlines manifest the oscillating nature for amplifying nonlinear mixed convection.

- Isotherms and isoconcentrations boundary layers enhance for raising values of thermal radiation.

\section{References}

1. Bejan A, A study of entropy generation in fundamental convective heat transfer. J Heat Transfer. 1979;101: 718-725.

2. Ali M, Khan WA, Irfan M, Sultan F, Shahzed M, Khan M. Computational analysis of entropy generation for cross nanofluid flow. Appl Nanosci. (2019).

3. Sheikholeslami M , Ganji DD. Entropy generation of nanofluid in presence of magnetic field using Lattice Boltzmann Method. Physica A. 2014;417: 273-286.

4. Khan MI, Kumar A, Hayat T, Waqas M, Singh R. Entropy generation in flow of Carreau nanofluid. J Mol Liq. 2019;278:677-687. 
5. Akbarzadeh M, Rashidi S, Keshmiri A, Shokri N. The optimum position of porous insert for a double-pipe heat exchanger based on entropy generation and thermal analysis. J Therm Anal Calorim. (2020); 139: 411-426.

6. Azadi M, Hosseinirad E, Hormozi F, Rashidi N. Second law analysis for nanofluid flow in mini-channel heat sink with finned surface: a study on fin geometries. J Therm Anal Calorim. (2020); 140: 1883-1895.

7. Shamsabadi H, Rashidi S, Esfahani JA. Entropy generation analysis for nanofluid flow inside a duct equipped with porous baffles. J Therm Anal Calorim. (2019); 135: 10091019.

8. Darbari B, Rashidi S, Keshmiri A. Nanofluid heat transfer and entropy generation inside a triangular duct equipped with delta winglet vortex generators. J Therm Anal Calorim. (2020);140: 1045-1055.

9. Eegunjobi AS, Makinde OD. Irreversibility Analysis of MHD Buoyancy-Driven Variable Viscosity Liquid Film along an Inclined Heated Plate Convective Cooling. J Appl Comput Mech. (2019); 5: 840-848.

10. Chamkha AJ, Selimefendigil F, Oztop HF. MHD Mixed Convection and Entropy Generation in a Lid-Driven Triangular Cavity for Various Electrical Conductivity Models. Entropy. (2018); 20: 903.

11. Selimefendigil F, Oztop HF. Effects of conductive curved partition and magnetic field on natural convection and entropy generation in an inclined cavity filled with nanofluid. Physica A. (2020); 540: 123004

12. Alsabery AI, Selimefendigil F, Hashim I, Chamkha AJ, Ghalambaz M. Fluid-structure interaction analysis of entropy generation and mixed convection inside a cavity with flexible right wall and heated rotating cylinder. Int J Heat Mass Tran. (2019); 140: 331-345.

13. Selimefendigil F, Oztop HF, Chamkha AJ. MHD mixed convection and entropy generation of nanofluid filled lid driven cavity under the influence of inclined magnetic fields imposed to its upper and lower diagonal triangular domains. J Magn Magn Mater. (2016);406:266-281.

14. Selimefendigil F, Oztop HF. MHD mixed convection and entropy generation of power law fluids in a cavity with a partial heater under the effect of a rotating cylinder. Int $\mathrm{J}$ Heat Mass Tran. (2016); 98: 40-51.

15. Basha HT, Sivaraj R, Reddy AS, Chamkha AJ,Tilioua M. Impacts of temperaturedependent viscosity and variable Prandtl number on forced convective FalknerSkan flow of Williamson nanofluid. SN Appl Sci.(2020): 2;477.

16. Kumar BR, Sivaraj R. Heat and mass transfer in MHD viscoelastic fluid flow over a vertical cone and flat plate with variable viscosity. Int J Heat Mass Transf. 2013;56: 370-379.

17. Mythili D, Sivaraj R, Influence of higher order chemical reaction and non-uniform heat source / sink on Casson fluid flow over a vertical cone and flat plate. J Mol Liq. 2016;216: 466-475.

18. Gaffar SA, Ur-rehman K, Reddy PR, Prasad VR, Khan BH. Powell-Eyring fluid flow towards an isothermal sphere in a non-darcy porous medium. Can J Phys. 2019;97: 1039-1048.

19. Animasaun IL, Mahanthesh B, Sarojamma G, Damisa JS. Significance of thickness of paraboloid of revolution and buoyancy forces on the dynamics of Erying Powell fluid subject to equal diffusivity kind of quartic autocatalysis. Physica A. 2020; 124047.

20. Sivaraj R, Benazir AJ, Srinivas S, Chamkha AJ. Investigation of cross-diffusion effects on Casson fluid flow in existence of variable fluid properties. Eur Phys J Spec Top. 2019;228: 35-53.

21. Reddy GJ, Kumar M, Beg OA. Effect of temperature dependent viscosity on entropy generation in transient viscoelastic polymeric fluid flow from an isothermal vertical plate. Physica A. 2018;510:426-445.

22. Prasad VR, Kumar BR. Non-Similar Comutational Solutions for Double-Di usive MHD Transport Phenomena for Non-Newtnian Nanofluid From a Horizontal Circular Cylinder. Nonlinear Eng. 2019;8:470-485.

23. Gaffar SA, Prasad VR, Beg OA, Numerical study of flow and heat transfer of nonNewtonian Tangent Hyperbolic fluid from a sphere with Biot number effects. Alexandria Eng J. 2015; 54: 829-841. 
24. Khan MI, Khan TA, Qayyum S, Hayat T, Khan MI, Alsaedi A. Entropy generation optimization and activation energy in nonlinear mixed convection flow of a tangent hyperbolic nanofluid. Eur Phys J Plus. 2018; 133:329.

25. Nayak MK, Sen SS, Shaw S, Makinde OD, Chamkha AJ. Investigation of Partial Slip and Viscous Dissipation Effects on the Radiative Tangent Hyperbolic Nanofluid Flow Past a Vertical Permeable Riga Plate with Internal Heating: Bungiorno Model. J Nanofluid. 2019; 1-12.

26. Vasu B, Gorla RSR, Beg OA, Murthy PVSN, Prasad VR, Kadir A. Unsteady Flow of a Nanofluid over a Sphere with Nonlinear Boussinesq Approximation. J Thermophys Heat Transf. 2019; 33:1-13.

27. Mahanthesh B, Gireesha BJ, Thammanna GT, Shehzad SA, Abbasi FM, Gorla RSR. Nonlinear convection in nano Maxwell fluid with nonlinear thermal radiation: A threedimensional study. Alexandria Eng J. 2018;57:1927-1935.

28. Waqas M, Dogonchi AS, Shehzad SA, Khan MI, Hayat T, Alsaedi A. Nonlinear convection and joule heating impacts in magneto-thixotropic nano fl uid strati fi ed fl ow by convectively heated variable thicked surface. J Mol Liq. 2020;300: 111945.

29. Patil PM, Shashikant A, Hiremath PS. Diffusion of liquid hydrogen and oxygen in nonlinear mixed convection nanofluid flow over vertical cone. Int J Hydrogen Energy. 2019;44:17061-17071.

30. Kameswaran PK, Sibanda P, Partha MK, Murthy PVSN. Thermophoretic and Nonlinear Convection in Non-Darcy Porous Medium. J Heat Transfer. 2015;136: 1-9.

31. Choi SUS, and Eastman JA, Enhancing thermal conductivity of fluids with nanoparticles. In: Proceedings of the 1995 ASME international mechanical engineering congress and exposition, 1217 November (American Society of Mechanical Engineers, fluids engineering division (publication) FED, 1995). (1995); 231: 99-105.

32. Nazari S, Ellahi R, Sarafraz MM, Safaei MR, Asgari A, Akbari OA. Numerical study on mixed convection of a non-Newtonian nanofluid with porous media in a two lid-driven square cavity. J Therm Anal Calorim. (2020);140:1121-1145.

33. Khan LA, Raza M, Mir NA, Ellahi R. Effects of different shapes of nanoparticles on peristaltic flow of MHD nanofluids filled in an asymmetric channel. J Therm Anal Calorim. (2020); 140: 879-890.

34. Hong K, Yang Y, Rashidi S, Guan Y, Xiong Q. Numerical simulations of a Cuwater nanofluid-based parabolic-trough solar collector. J Therm Anal Calorim. (2020); https://doi.org/10.1007/s10973-020-09386-4.

35. Raza M, Ellahi R, Sait, SM, Sarafraz MM, Shadloo MS, Waheed I. Enhancement of heat transfer in peristaltic flow in a permeable channel under induced magnetic field using different CNTs. J Therm Anal Calorim. (2020):140; 1277-1291.

36. Akar S, Rashidi S, Esfahani JA, Karimi N. Targeting a channel coating by using magnetic field and magnetic nanofluids. J Therm Anal Calorim. (2019);137: 381-388.

37. Tiwari RJ, Das MK. Heat transfer augmentation in a two-sided lid-driven differentially heated square cavity utilizing nanofluids. Int. J. Heat. Mass Transf., (2007); 50:20022018.

38. Buongiorno J. Convective transport in nanofluids. J Heat Transfer. (2006); 128:240.

39. Rashad AM, Chamkha AJ, Modather M. Mixed convection boundary-layer flow past a horizontal circular cylinder embedded in a porous medium filled with a nanofluid under convective boundary condition. Comput Fluids. 2013;86: 380-388.

40. Merkin JH. Free convection boundary layers on cylinders of elliptic cross section, J Heat Transfer. 1977;99: 453-457.

41. Yih KA. Effect of blowing/suction on MHD-natural convection over horizontal cylinder: UWT or UHF, Acta Mech. 2000;144: 17-27.

42. Cebeci T, Pradshaw P. Physical and Computational Aspects of Convective Heat Transfer,Springer,New York, 1998. 
Table 3 Impacts of $N_{r}$ and $E_{c}$ on local skin friction coefficient $\left(C_{f}^{*}\right)$, dimensionless local rate of heat transfer $\left(N u^{*}\right)$ and dimensionless local rate of mass transfer $\left(S h^{*}\right)$ for various values of $\xi$.

\begin{tabular}{|c|c|c|c|c|c|c|c|c|c|}
\hline \multirow{2}{*}{$\begin{array}{c}\text { Physical } \\
\text { Parameters }\end{array}$} & \multirow[t]{2}{*}{ Values } & \multirow{2}{*}{$\begin{array}{l}\text { Physical } \\
\text { Quantities }\end{array}$} & & \multirow{2}{*}{$\begin{array}{c}\text { CPU } \\
\text { time(Sec.) }\end{array}$} \\
\hline & & & 0 & 0.2 & 0.4 & 0.6 & 0.8 & 1.0 & \\
\hline \multirow{12}{*}{$N_{r}$} & \multirow{3}{*}{0.1} & $C_{f}^{*}$ & 0 & 0.3984 & 0.7626 & 1.0709 & 1.3065 & 1.4599 & \multirow{3}{*}{11.438911} \\
\hline & & $\frac{J}{N u^{*}}$ & 1.1194 & 1.1090 & 1.0884 & 1.0580 & 1.0185 & 0.9706 & \\
\hline & & $S h^{*}$ & 0.8418 & 0.8354 & 0.8226 & 0.8036 & 0.7784 & 0.7473 & \\
\hline & \multirow{3}{*}{0.3} & $C_{f}^{*}$ & 0 & 0.3829 & 0.7321 & 1.0261 & 1.2489 & 1.3907 & \multirow{3}{*}{11.858886} \\
\hline & & $N u^{*}$ & 1.1146 & 1.1042 & 1.0835 & 1.0529 & 1.0131 & 0.9649 & \\
\hline & & $S h^{*}$ & 0.8356 & 0.8291 & 0.8161 & 0.7968 & 0.7712 & 0.7395 & \\
\hline & \multirow{3}{*}{0.5} & $C_{f}^{*}$ & 0 & 0.3672 & 0.7013 & 0.9810 & 1.1906 & 1.3208 & \multirow{3}{*}{11.161408} \\
\hline & & $N u^{*}$ & 1.1097 & 1.0992 & 1.0783 & 1.0475 & 1.0075 & 0.9589 & \\
\hline & & $S h^{*}$ & 0.8291 & 0.8225 & 0.8093 & 0.7897 & 0.7636 & 0.7314 & \\
\hline & \multirow{3}{*}{0.7} & $C_{f}^{*}$ & 0 & 0.3515 & 0.6703 & 0.9356 & 1.1318 & 1.2502 & \multirow{3}{*}{11.189030} \\
\hline & & $N u^{*}$ & 1.1046 & 1.0940 & 1.0730 & 1.0420 & 1.0016 & 0.9527 & \\
\hline & & $S h^{*}$ & 0.8225 & 0.8158 & 0.8023 & 0.7823 & 0.7557 & 0.7228 & \\
\hline \multirow{12}{*}{$E_{c}$} & \multirow{3}{*}{0.01} & $C_{f}^{*}$ & 0 & 0.3984 & 0.7626 & 1.0709 & 1.3065 & 1.4599 & \multirow{3}{*}{11.438911} \\
\hline & & $N u^{*}$ & 1.1194 & 1.1090 & 1.0884 & 1.0580 & 1.0185 & 0.9706 & \\
\hline & & $S h^{*}$ & 0.8418 & 0.8354 & 0.8226 & 0.8036 & 0.7784 & 0.7473 & \\
\hline & \multirow{3}{*}{0.1} & $C_{f}^{*}$ & 0 & 0.3674 & 0.7021 & 0.9833 & 1.1952 & 1.3288 & \multirow{3}{*}{11.072150} \\
\hline & & $N u^{*}$ & 1.1097 & 1.0888 & 1.0475 & 0.9885 & 0.9154 & 0.8332 & \\
\hline & & $S h^{*}$ & 0.8291 & 0.8230 & 0.8108 & 0.7926 & 0.7682 & 0.7378 & \\
\hline & \multirow{3}{*}{0.3} & $C_{f}^{*}$ & 0 & 0.3677 & 0.7038 & 0.9883 & 1.2057 & 1.3468 & \multirow{3}{*}{11.256386} \\
\hline & & $N u^{*}$ & 1.1097 & 1.0656 & 0.9786 & 0.8558 & 0.7068 & 0.5453 & \\
\hline & & $S h^{*}$ & 0.8291 & 0.8241 & 0.8142 & 0.7991 & 0.7787 & 0.7525 & \\
\hline & \multirow{3}{*}{0.5} & $C_{f}^{*}$ & 0 & 0.3680 & 0.7056 & 0.9934 & 1.2163 & 1.3653 & \multirow{3}{*}{11.169868} \\
\hline & & $N u^{*}$ & 1.1097 & 1.0424 & 0.9092 & 0.7210 & 0.4927 & 0.2452 & \\
\hline & & $S h^{*}$ & 0.8291 & 0.8252 & 0.8175 & 0.8057 & 0.7894 & 0.7677 & \\
\hline
\end{tabular}


Table 4 Impacts of $N_{T}$ and $N_{B}$ on local skin friction coefficient $\left(C_{f}^{*}\right)$, dimensionless local rate of heat transfer $\left(N u^{*}\right)$ and dimensionless local rate of mass transfer $\left(S h^{*}\right)$ for various values of $\xi$.

\begin{tabular}{|c|c|c|c|c|c|c|c|c|c|}
\hline \multirow{2}{*}{$\begin{array}{c}\text { Physical } \\
\text { Parameters }\end{array}$} & \multirow[t]{2}{*}{ Values } & \multirow{2}{*}{$\begin{array}{c}\text { Physical } \\
\text { Quantities }\end{array}$} & \multicolumn{6}{|c|}{$\zeta$} & \multirow{2}{*}{$\begin{array}{c}\text { CPU } \\
\text { time(Sec.) }\end{array}$} \\
\hline & & & 0 & 0.2 & 0.4 & 0.6 & 0.8 & 1.0 & \\
\hline \multirow{12}{*}{$N_{T}$} & \multirow{3}{*}{0.01} & $C_{f}^{*}$ & 0 & 0.3675 & 0.7019 & 0.9819 & 1.1917 & 1.3221 & \multirow{3}{*}{10.986395} \\
\hline & & $N u^{*}$ & 1.1192 & 1.1086 & 1.0876 & 1.0566 & 1.0162 & 0.9673 & \\
\hline & & $S h^{*}$ & 0.8428 & 0.8361 & 0.8226 & 0.8025 & 0.7760 & 0.7431 & \\
\hline & \multirow{3}{*}{0.1} & $C_{f}^{*}$ & 0 & 0.3672 & 0.7013 & 0.9810 & 1.1906 & 1.3208 & \multirow{3}{*}{11.788723} \\
\hline & & $N u^{*}$ & 1.1097 & 1.0992 & 1.0783 & 1.0475 & 1.0075 & 0.9589 & \\
\hline & & $S h^{*}$ & 0.8291 & 0.8225 & 0.8093 & 0.7897 & 0.7636 & 0.7314 & \\
\hline & \multirow{3}{*}{0.3} & $C_{f}^{*}$ & 0 & 0.3666 & 0.7001 & 0.9793 & 1.1884 & 1.3183 & \multirow{3}{*}{11.129852} \\
\hline & & $N u^{*}$ & 1.0889 & 1.0786 & 1.0581 & 1.0278 & 0.9884 & 0.9407 & \\
\hline & & $S h^{*}$ & 0.8016 & 0.7953 & 0.7826 & 0.7637 & 0.7387 & 0.7077 & \\
\hline & \multirow{3}{*}{0.5} & $C_{f}^{*}$ & 0 & 0.3661 & 0.6991 & 0.9778 & 1.1865 & 1.3160 & \multirow{3}{*}{10.991573} \\
\hline & & $N u^{*}$ & 1.0687 & 1.0585 & 1.0384 & 1.0086 & 0.9699 & 0.9229 & \\
\hline & & $S h^{*}$ & 0.7778 & 0.7717 & 0.7595 & 0.7414 & 0.7172 & 0.6872 & \\
\hline \multirow{12}{*}{$N_{B}$} & \multirow{3}{*}{0.3} & $C_{f}^{*}$ & 0 & 0.3672 & 0.7013 & 0.9810 & 1.1906 & 1.3208 & \multirow{3}{*}{10.967421} \\
\hline & & $N u^{*}$ & 1.1097 & 1.0992 & 1.0783 & 1.0475 & 1.0075 & 0.9589 & \\
\hline & & $S h^{*}$ & 0.8291 & 0.8225 & 0.8093 & 0.7897 & 0.7636 & 0.7314 & \\
\hline & \multirow{3}{*}{0.5} & $C_{f}^{*}$ & 0 & 0.3679 & 0.7026 & 0.9829 & 1.1929 & 1.3236 & \multirow{3}{*}{11.181454} \\
\hline & & $N u^{*}$ & 1.0769 & 1.0667 & 1.0465 & 1.0166 & 0.9777 & 0.9306 & \\
\hline & & $S h^{*}$ & 0.8369 & 0.8303 & 0.8169 & 0.7971 & 0.7708 & 0.7382 & \\
\hline & \multirow{3}{*}{0.7} & $C_{f}^{*}$ & 0 & 0.3684 & 0.7035 & 0.9843 & 1.1947 & 1.3258 & \multirow{3}{*}{11.330983} \\
\hline & & $N u^{*}$ & 1.0449 & 1.0350 & 1.0153 & 0.9863 & 0.9485 & 0.9028 & \\
\hline & & $S h^{*}$ & 0.8405 & 0.8337 & 0.8204 & 0.8004 & 0.7740 & 0.7414 & \\
\hline & \multirow{3}{*}{0.9} & $C_{f}^{*}$ & 0 & 0.3688 & 0.7045 & 0.9856 & 1.1964 & 1.3277 & \multirow{3}{*}{11.356811} \\
\hline & & $N u^{*}$ & 1.0136 & 1.0040 & 0.9849 & 0.9567 & 0.9200 & 0.8756 & \\
\hline & & $S h^{*}$ & 0.8425 & 0.8358 & 0.8224 & 0.8024 & 0.7760 & 0.7433 & \\
\hline
\end{tabular}

